\title{
A GENERALIZED BERELE-SCHENSTED ALGORITHM AND CONJECTURED YOUNG TABLEAUX FOR INTERMEDIATE SYMPLECTIC GROUPS
}

\author{
ROBERT A. PROCTOR
}

\begin{abstract}
The Schensted and Berele algorithms combinatorially mimic the decompositions of $\otimes^{k} V$ with respect to $\mathrm{GL}_{N}$ and $\mathrm{Sp}_{2 n}$. Here we present an algorithm which is a common generalization of these two algorithms. "Intermediate symplectic groups" $\mathrm{Sp}_{2 n, m}$ are defined. These groups interpolate between $\mathrm{GL}_{N}$ and $\mathrm{Sp}_{N}$. We conjecture that there is a decomposition of $\otimes^{k} V$ with respect to $\mathrm{Sp}_{2 n, m}$ which is described by the output of the new algorithm.
\end{abstract}

\section{INTRODUCTION}

Half of the motivation for this mostly combinatorial paper comes from representation theory. However, no knowledge of representation theory is needed to understand it, except for $\S 7$ and the motivation given in this introduction. The heart of the paper, $\S \S 3-6$, will be most easily read by someone familiar with Schensted's algorithm or Schützenberger's theory of jeu d'tacquin.

In 1961 Schensted invented an algorithm for finding the longest increasing subsequence of a sequence of numbers [Sch]. Since then it has become well known that when this algorithm is applied to a certain set of inputs, the pairs of tableaux output by the algorithm are the same as the pairs of tableaux which arise in the combinatorial description of a famous representation decomposition found by Schur and Weyl. That decomposition is of the $\mathrm{GL}_{m}$-module $\otimes^{k} V$, which is formed by starting with the action of $\mathrm{GL}_{m}$ on $V=\mathrm{C}^{m}$. One can instead start with $\mathrm{Sp}_{2 n}$ acting on $V=\mathrm{C}^{2 n}$ and then again decompose $\otimes^{k} V$. In 1985 Berele found an algorithm similar to Schensted's which combinatorially "mimicked" the decomposition of this symplectic tensor representation in a manner analogous to the "mimicking" by Schensted of the general linear decomposition.

Received by the editors January 26, 1988 and, in revised form, March 17, 1989.

1980 Mathematics Subject Classification (1985 Revision). Primary 05A17, 05A19, 22E46.

Key words and phrases. Schensted's algorithm, jeu d'tacquin, symplectic groups, tensor representations of Lie groups, Schur functions, group characters.

Supported by an NSF Mathematical Sciences Postdoctoral Fellowship.

These results have been presented at the Annual Meeting of the American Mathematical Society, San Antonio, January 1987, in a special session on Combinatorics and group representations. 
In the first half of this paper we develop a common generalization of the Schensted and Berele algorithms inspired by the notion of "intermediate symplectic group $\mathrm{Sp}_{2 n, m}$ ", which acts on $\mathbf{C}^{2 n+m}$. Our algorithm is in a sense a finer version of Berele's algorithm. In the two extreme special cases of $n=0$ and $m=0$, this new algorithm immediately reduces to the Schensted and Berele algorithms respectively. In the second half of the paper we will study constructions in the subjects of symmetric functions and representation theory which parallel the generalized algorithm. Reasoning by analogy we conjecture that there is a decomposition of $\otimes^{k} V$ for $\operatorname{Sp}_{2 n, m}$ (where now $V=\mathrm{C}^{2 n+m}$ ) which is described by the output of the generalized algorithm. Although we do not give any direct connections between our algorithm and representation theory, we hope that the conjecture and its supporting evidence will at least serve as an advertisement for the remarkable agreement between the theory of jeu d'tacquin and the theory of tensor representations. Two byproducts of this work which are independent of representation theory and of the symplectic combinatorial environment are the notions of marked jeu d'tacquin ( $(3)$ and marked skew Schur function (Lemma 6.1). Perhaps these constructions will arise elsewhere.

Let

$$
B_{2 n, m}=\left(\begin{array}{ccccccccc}
0 & 1 & & & & & & & \\
-1 & 0 & & & & & & & \\
& & \ldots & & & & & & \\
& & & 0 & 1 & & & & \\
& & & -1 & 0 & & & & \\
& & & & & 0 & & & \\
& & & & & & 0 & & \\
& & & & & & & \ldots & \\
& & & & & & & 0
\end{array}\right) \text {, }
$$

be a $(2 n+m) \times(2 n+m)$ matrix with the usual bilinear form for $\mathrm{Sp}_{2 n}$ in the upper left corner. Define the intermediate symplectic group $\mathrm{Sp}_{2 n, m}$ to be the subgroup of $\mathrm{GL}_{2 n+m}$ which fixes $B_{2 n, m}$ as a bilinear form. When $n>0$ and $m>0$ this group is not reductive:

$$
\mathrm{Sp}_{2 n, m}=\left(\begin{array}{cc}
\mathrm{Sp}_{2 n} & 0 \\
* & \mathrm{GL}_{m}
\end{array}\right)
$$

Obviously $\mathrm{Sp}_{2 n, m}=\mathrm{GL}_{m}$ when $n=0$ and $\mathrm{Sp}_{2 n, m}=\mathrm{Sp}_{2 n}$ when $m=$ 0 . The most interesting truly intermediate case is the odd symplectic group $\mathrm{Sp}_{2 n+1}:=\mathrm{Sp}_{2 n, 1}$. Elsewhere we have shown [Pr1] that the dimensions and characters of "trace-free" tensor representations of the groups $\mathrm{Sp}_{2 n+1}$ nicely fill a series of gaps in a framework formed by analogous quantities for the classical groups $\mathrm{Sp}_{2 n}, \widetilde{\mathrm{SO}}_{2 n}$, and $\widetilde{\mathrm{SO}}_{2 n+1}$. The denominator of the character formula for tracefree representations of $\mathrm{Sp}_{2 n+1}$ can be written [Pr1] as a product over the nonreduced root system $B C_{n}$. Understanding the tensor representations of these potentially important new Lie groups $\mathrm{Sp}_{2 n+1}$ is one of the main motivations for this paper. But most of our results will hold for the more general 
$\mathrm{Sp}_{2 n, m}$. Fix $N>0$. The $\mathrm{Sp}_{2 n, m}$ for $N=2 n+m$ interpolate between $\mathrm{GL}_{N}$ and $\mathrm{Sp}_{2 n}$ or $\mathrm{Sp}_{2 n+1}$ :

$$
\begin{gathered}
\mathrm{GL}_{N}=\mathrm{Sp}_{0, N} \supset \mathrm{Sp}_{2, N-2} \supset \mathrm{Sp}_{4, N-4} \supset \cdots \supset \\
\supset \mathrm{Sp}_{2 n, 0}=\mathrm{Sp}_{2 n} \text { or } \supset \mathrm{Sp}_{2 n, 1}=\mathrm{Sp}_{2 n+1},
\end{gathered}
$$

depending upon whether $N$ is even or odd.

There are three parallel contexts in which the matters at hand can be viewed: combinatorial-algorithmic ( $\S 2-5)$, representation theoretic $(\S 7)$, and symmetric function theoretic ( $\$ \S 6,8$ and 9$)$. In this paper our treatment of the third context is very concrete and does not use the symmetry of the polynomials at hand; so henceforth we will usually refer to the third context as that of generating functions, or polynomial theoretic. Recall (e.g. [Mac]) that the Schur function $s_{\lambda}\left(z_{1}, z_{2}, \ldots, z_{m}\right)$ can be expressed as the sum of certain weight monomials $z(T)$ over the set of all semistandard Young tableaux of shape $\lambda$. (Consult $\S 2$ for more detailed definitions of this and other terminology below.) Let $f_{\lambda}$ be the number of standard Young tableaux of shape $\lambda$. Consider the polynomial identity

$$
\left(z_{1}+z_{2}+\cdots+z_{m}\right)^{k}=\sum f_{\lambda} s_{\lambda}\left(z_{1}, z_{2}, \ldots, z_{m}\right),
$$

where the sum is over all shapes $\lambda$ with $k$ squares and no more than $m$ rows. This identity can be interpreted in each of the three contexts. For the algorithmic interpretation, the left-hand side can be viewed as listing all possible Schensted input "words" of length $k$ from the alphabet $\{1,2, \ldots, m\}$. The right-hand side describes the collection of outputs which result from applying the algorithm to this collection of inputs: Each output is a pair of tableaux $(P, Q)$ each of the same shape $\lambda$, where $P$ is semistandard and $Q$ is standard. Collecting the output pairs according to shape gives the right-hand side. The main result of the original Schensted paper was that Schensted's algorithm gives a bijection between the "left-hand" and "right-hand" collections of objects just described. So the identity above can be viewed as an immediate consequence of the main fact concerning Schensted's algorithm. This identity can also be proved with representation theory. Let $V=C^{m}$, and decompose $\otimes^{k} V$ into $\mathrm{GL}_{m}$ irreducibles. Take the $\mathrm{GL}_{m}$ characters of both sides of this decomposition. To get the right-hand side, combine the following two facts with the Schur double centralizer theorem: $s_{\lambda}\left(z_{1}, z_{2}, \ldots, z_{m}\right)$ is the character of the $\lambda$ th irreducible representation of $\mathrm{GL}_{m}$, and $f_{\lambda}$ is the dimension of the $\lambda$ th irreducible representation of the symmetric group $S_{k}$. Lastly, there is a simple proof of (1.1) in the polynomial context which uses only easy determinant calculations. This proof can be easily written down while reading the proof of Theorem 9.1(a) of this paper. With respect to the " $2 n+m$ " generality of this paper, all of the preceding is the special case $n=0$.

Now consider the $m=0$ analogs of the above observations. For the purposes of this introduction, define $\operatorname{sp}_{\mu}\left(y_{1}, y_{2}, \ldots, y_{n}\right)$ to be the character of the $\mu$ th 
irreducible representation of $\mathrm{Sp}_{2 n}$. There is a combinatorial description of this (Laurent) polynomial due to Zhelobenko and King which sums over a special kind ("symplectic") of semistandard tableaux. Note that $y_{1}+y_{1}^{-1}+y_{2}+y_{2}^{-1}+$ $\cdots+y_{n}+y_{n}^{-1}$ is the character of $\mathrm{Sp}_{2 n}$ acting on $V=\mathbf{C}^{2 n}$. Berele gave [Be1] two proofs of the following character or polynomial identity:

$$
\left(y_{1}+y_{1}^{-1}+y_{2}+y_{2}^{-1}+\cdots+y_{n}+y_{n}^{-1}\right)^{k}=\sum g_{\mu}(k, n) \operatorname{sp}_{\mu}\left(y_{1}, y_{2}, \ldots, y_{n}\right) \text {. }
$$

Here the sum is over all shapes $\mu$ with $k-2 l$ squares, $l \geq 0$, and no more than $n$ rows, and $g_{\mu}(k, n)$ is a number related to $f_{\lambda}$ which is defined combinatorially at the end of $\S 2$. Although Berele's first proof as stated was a mixture of representation theory and polynomial calculations, it was in essence a polynomial proof. (Our proof of Theorem 9.1(a) restates that proof in a complete, simple and explicitly pure polynomial form.) The second proof of this identity in [Be1] was obtained by writing down the generating function version of the main result, viz. the bijective property of Berele's algorithm.

To obtain identity (1.2) algorithmically, one inputs all words of length $k$ from the alphabet $\{1, \overline{1}, 2, \overline{2}, \ldots, n, \bar{n}\}$ into Berele's $\mathrm{Sp}_{2 n}$ algorithm. As the left tableaux is being constructed, sometimes a letter $i$ will annihilate a letter $\bar{i}$. This reflects $y_{i} y_{i}^{-1}=1$ in the polynomial context. Our extension of Berele's algorithm is designed to handle words from the alphabet $\{1, \overline{1}, 2, \overline{2}, \ldots, n, \bar{n}$, $n+1, n+2, \ldots, n+m\}$, wherein the first $2 n$ letters occur in inverse pairs as with Berele's algorithm and the last $m$ letters occur without inverses as in Schensted's algorithm. The main result of this paper, Theorem 5.1, describes the output that results when all words of length $k$ from this alphabet are input. Identity (1.3) below is the generating function version of Theorem 5.1. The "intermediate symplectic Schur functions" $\operatorname{sp}_{\lambda, \mu}\left(y_{1}, \ldots, y_{n} ; z_{1}, \ldots, z_{m}\right)$ and the numbers $h_{\lambda, \mu}(k, n, m)$ appearing in this identity are combinatorially defined in $\S 2$ :

$$
\begin{aligned}
& \left(y_{1}+y_{1}^{-1}+y_{2}+y_{2}^{-1}+\cdots+y_{n}+y_{n}^{-1}+z_{1}+\cdots+z_{m}\right)^{k} \\
& \quad=\sum h_{\lambda, \mu}(k, n, m) \operatorname{sp}_{\lambda, \mu}\left(y_{1}, \ldots, y_{n} ; z_{1}, \ldots, z_{m}\right) .
\end{aligned}
$$

Here the sum is over all " $n$-marked shapes" $(\lambda, \mu)$ with $k-2 l$ squares, $l \geq 0$, and with no more than $n+m$ rows. When $n=0$ and $m=0$ this identity immediately reduces to identities (1.1) and (1.2) respectively. For general $m>$ 0 the algorithmic approach of Theorem 5.1 is the only known proof of (1.3). Theorem 9.1 provides simple polynomial proofs of $(1.3)$ in the cases $m=$ 0 (i.e. the $\mathrm{Sp}_{2 n}$ case (1.2)) and $m=1$ (i.e. the $\mathrm{Sp}_{2 n+1}$ case). Given the agreement between the algorithms and representation theory in the $n=0$ and $m=0$ cases, it is natural to hope that the correspondence continues. Conjecture 7.1 describes how the output of our "marked Berele" algorithm is expected to describe a nice decomposition of $\otimes^{k} V$ with respect to $\mathrm{Sp}_{2 n, m}$, where $V=$ $\mathbf{C}^{2 n+m}$. 
Most of the results in this paper depend upon the marked jeu d'tacquin construction, which is developed in $\S 3$. This is a generalization of Schutzenberger's jeu d'tacquin construction wherein now every square in the plane is marked or unmarked, and the region of unmarked squares forms a "shape" in the upper left corner. In $\S 4$ we present our extension of Berele's algorithm and largely formulate the new algorithm in terms of marked jeu d'tacquin. The main result, viz. an extension of Berele's bijection, appears in $\S 5$. The hardest part of the development was finding the correct characterization of the output of the algorithm. The well-definedness of marked jeu d'tacquin is the key fact needed for the proof of the main result Theorem 5.1.

All combinatorial definitions have been placed in $\S 2$. The main result, Theorem 5.1 , should be read first. Then read $\S \S 3-5$ while referring to $\S 2$ as necessary. The rest of the paper consists of three independent parts. $\S 6$ uses combinatorial techniques to obtain symmetric function results concerning the $\mathrm{sp}_{\lambda, \mu} . \S 7 \mathrm{de}-$ scribes the conjectured relationship with representations of $\mathrm{Sp}_{2 n, m}, \S \S 8$ and 9 present elementary proofs of (1.2) and the $m=1$ case of (1.3). The remainder of the introduction consists of miscellaneous comments which should only be glanced at during a first reading.

$\S \S 8$ and 9 were written in greater generality than needed for $\mathrm{Sp}_{N}$ with possible future application to the orthogonal groups $\mathrm{O}_{N}$ in mind. Since the original version of this paper was written, the same kind of reasoning that led to Theorem 5.1 was combined with the kinds of techniques used in $\S \S 8$ and 9 to simultaneously discover the "correct" semistandard tableaux for irreducible representations of $\mathrm{O}_{N}[\mathrm{Pr} 4]$ and an analog of Berele's algorithm for tensor representations of $\mathrm{O}_{N}$ [Pr3]. So although the connection between this kind of algorithm and representation theory is not at all understood, the algorithm viewpoint has been applied to representation theory at least as a heuristic tool to help find the "correct" orthogonal tableaux. Using these orthogonal tableaux, the proofs of $\S \S 8$ and 9 can now be applied almost immediately to obtain an orthogonal analog of (1.2).

From the point of view of the theory of symmetric functions, the characters $\operatorname{sp}_{\lambda}\left(y_{1}, \ldots, y_{n}\right)$ can be viewed as close relatives to the Schur functions $s_{\lambda}\left(z_{1}, \ldots, z_{n}\right)$. In fact, if $\lambda$ is a partition of $k$, then the sum of the terms of $\operatorname{sp}_{\lambda}\left(y_{1}, \ldots, y_{n}\right)$ of degree $k$ is $s_{\lambda}\left(y_{1}, \ldots, y_{n}\right)$. But now $\operatorname{sp}_{\lambda}\left(y_{1}, \ldots, y_{n}\right)$ is invariant under the interchange $y_{i} \leftrightarrow y_{i}^{-1}$ as well as $y_{i} \leftrightarrow y_{j}$. More generally, Proposition 6.1 states that $\operatorname{sp}_{\lambda, \mu}\left(y_{1}, \ldots, y_{n} ; z_{1}, \ldots, z_{m}\right)$ simultaneously has this $S_{n} \times \mathbf{Z}_{2}^{n}$ symmetry in the $y$ variables together with $S_{m}$ symmetry in the $z$ variables. Hence the intermediate symplectic Schur functions form a new class of generalized symmetric functions. The definition of the $\operatorname{sp}_{\lambda, \mu}(y ; z)$ shows that the $y$ variables and the $z$ variables are joined together in a nonobvious and nontrivial fashion involving jeu d'tacquin. These symmetry properties and the decomposition property stated in Proposition 6.2 are properties that one would expect of characters of $\mathrm{Sp}_{2 n, m}$. A side development 
in $\S 6$ is the notion of "marked skew Schur function" which can be regarded as a "sub-" skew Schur function in the following sense: If the ordinary skew Schur function $s_{\lambda / \theta}(z)=\sum b_{\nu} s_{\nu}(z)$, then the marked skew Schur function $s_{\lambda / \theta, \mu}(z)=\sum b_{\nu}^{\prime} s_{\nu}(z)$ with $0 \leq b_{\nu}^{\prime} \leq b_{\nu}$.

Even in the nonintermediate case of $\operatorname{Sp}_{2 n}$ (i.e. $m=0$ ), the representation theoretic situation is not well understood. At present there is no known purely representation theoretic proof of (1.2) analogous to the purely algebraic proof of (1.1). (That proof does not use any character calculations, but uses Young symmetrizing projections to explicitly construct a basis for the tensor space which is indexed by pairs of tableaux $(P, Q)$.) Propositions 7.1 to 7.4 provide a good deal of supporting evidence for Conjecture 7.1 from several special cases. At present the conjecture does not uniquely characterize the proposed decomposition up to equivalence, but it would nonetheless be a remarkable agreement between combinatorics and representation theory if there was at least one such decomposition. In order to prove this conjecture, the relationship between jeu d'tacquin and representation theory must be understood at a deeper level: Unlike the $\mathrm{GL}_{N}$ and $\mathrm{Sp}_{2 n}$ cases, the jeu d'tacquin operation is an explicit part of the statement of the decomposition (via the definition of the $\operatorname{sp}_{\lambda, \mu}(y ; z)$ ).

Hopefully a proof of Conjecture 7.1 would lead to a purely representation theoretic understanding even of the $m=0$ identity (1.2). In his book, The Classical Groups, Weyl partly kept track of copies of the bilinear form B defining $\mathrm{Sp}_{2 n}$ when decomposing $\otimes^{k} V$ with respect to $\mathrm{Sp}_{2 n} . \S 7$ is a continuation of this approach to the $\mathrm{Sp}_{2 n, m}$ case. (In particular, read the paragraph between Propositions 7.3 and 7.4.) In contrast with the familiar $\mathrm{Sp}_{2 n}$ situation, $\mathrm{Sp}_{2 n, m}$ is not reductive and not all tensor characters are known a priori. The main point of Conjecture 7.1 is that the intermediate symplectic Schur functions $\operatorname{sp}_{\lambda, \mu}(y ; z)$ should provide an appropriate notion of tensor character. Some heuristic remarks ("vertical dominoes") concerning the relationship between the algorithm and representation theory are made after Lemma 7.2. Hanlon and Wales $[\mathrm{H}-\mathrm{W}]$ have recently obtained some results which describe the structure of the "Brauer" centralizing algebra for $\mathrm{Sp}_{2 n}$ acting on $\bigotimes^{k} V$. It might be interesting to study the interaction between this paper and [H-W]: As one passes from $\mathrm{GL}_{N}$ to $\mathrm{Sp}_{2 n}$ via the $\mathrm{Sp}_{2 n, m}$ with $2 n+m=N$, the centralizing algebra grows from the group algebra of the symmetric group $S_{k}$ to the $k$ th Brauer algebra.

Propositions 8.1 and 9.1 give Giambelli-like determinantal expressions for certain $\operatorname{sp}_{\lambda, \mu}(y ; z)$. In the $\mathrm{Sp}_{2 n}$ case, the proof of Proposition 8.1 can be used to give a simple derivation of the Zhelobenko-King combinatorial description [Zhe, Kil] of $\mathrm{sp}_{\lambda}(y)$, if one takes this determinant formula (equation 3.4 of [Ki2]) for the character $\operatorname{sp}_{\lambda}(y)$ as a starting point. (That formula can be derived from Weyl's quotient character formula by the generating function methods given on pp. 233 and 243-244 of [Lit].) These determinantal formulas play a central role in $\S 9$, where easy determinant manipulations are used to prove the $m=0$ and $m=1$ cases of (1.3). 
The same method used to prove the well-definedness of marked jeu d'tacquin (Proposition 3.1) and the symmetry of marked skew Schur functions (Lemma 6.1) can be used as the main step in a modified version of Schützenberger's proof of the Littlewood-Richardson rule [Pr2]. An entirely different view of the Littlewood-Richardson rule is taken in Lemma 9.2. Here a general determinant identity is given which can be used as the main step for computing the tensor product of an arbitrary representation with the defining representation ("onebox") for orthogonal or symplectic groups. How far can this approach to the Littlewood-Richardson rule be pushed, even in just the case of ordinary Schur functions?

\section{Definitions}

An $(n+m)$-partition $\lambda$ of $k \geq 0$ is a sequence $\lambda_{1} \geq \lambda_{2} \geq \cdots \geq \lambda_{n+m} \geq 0$ of integers such that $\lambda_{1}+\lambda_{2}+\cdots+\lambda_{n+m}=k=:|\lambda|$. The $(n+m)$-shape associated to $\lambda$ is a diagram $\lambda$ which has $\lambda_{i}$ squares in its $i$ th row. Throughout this paper $\alpha_{j}$ and $\beta_{j}$ will be the numbers of squares in the $j$ th columns of the shapes $\lambda$ and $\mu$ respectively. Consult Figure 1(a) during the following definition. An $n$-marked $(n+m)$-shape $(\lambda, \mu)$ consists of an $(n+m)$-shape $\lambda$ and an $n$ shape $\mu$ contained in $\lambda$ such that $m \geq \alpha_{1}-\beta_{1} \geq \alpha_{2}-\beta_{2} \geq \alpha_{3}-\beta_{3} \geq \cdots$. The first $\beta_{j}$ squares in the $j$ th column of the shape $(\lambda, \mu)$ are unmarked squares; the remaining $\alpha_{j}-\beta_{j}$ squares are marked squares. So $\mu$ is the region of unmarked spaces. Note that all squares in rows $n+1$ to $n+m$ must be marked. In a certain sense the partition $\lambda$ is the sum of an unmarked and a marked partition. See Figure 8 for additional examples. Incidentally, there is a more elegant equivalent definition (which we will not use). If $(\lambda, \mu)$ is an $n$-marked $(n+m)$-shape, then necessarily $\mu$ consists of a subset of the parts in $\lambda$. So assuming that all of the parts of $\mu$ appear in $\lambda$, define an m-circled $(n+m)$-partition $(\lambda, \mu)$ to be an $(n+m)$-partition $\lambda$ in which the $m$ parts not appearing in $\mu$ have been circled. If there are both uncircled and circled parts of a given size, list the circled parts after the uncircled parts of that size.

Suppose that a shape $\lambda$ contains a smaller shape $\theta$. Then the skew shape $\lambda / \theta$ is obtained by removing the squares in the region $\theta$ from the diagram of shape $\lambda$. A skew $n$-marked $(n+m)$-shape $(\lambda / \theta, \mu)$ is just an $n$-marked $(n+m)$-shape $(\lambda, \mu)$ from which the squares in $\theta$ have been removed. Note

(a)

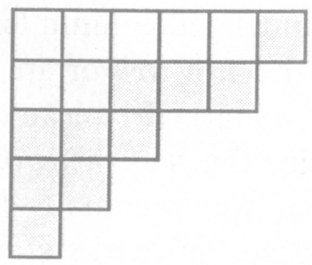

(b)

\begin{tabular}{|c|c|c|c|c|c|}
\hline 1 & 1 & 2 & 4 & 6 & 6 \\
\hline 3 & 5 & 5 & 5 & 7 & \\
\hline 5 & 6 & 6 & & & \\
\hline 6 & 7 & & & & \\
\hline 7 & & & & & \\
\hline
\end{tabular}

FigURE 1. $n=2, m=3 ; \lambda=(6,5,3,2,1)$; $\mu=(5,2) ; N=7$ 
that we do not require $\theta$ to be contained in $\mu$. When removing the squares of $\theta$, we do not remove any marks: Some marks will be said to extrude into the region $\theta$. (Glance at Figures 2-4.) For this reason it will sometimes be better to think in terms of the southeastern boundary of $\mu$ (e.g. the heavy line in the left hand part of Figure 5) or the column lengths $\beta_{j}$ of $\mu$ rather than the shape or region of $\mu$. In fact we will sometimes allow the column lengths $\beta_{j}$ to become negative, but we will still require $n \geq \beta_{1} \geq \beta_{2} \geq \cdots$. If $\beta_{j}<0$, then all $\alpha_{j}$ squares in the $j$ th column of the shapes $\theta$ and $\lambda$ are marked, and in addition $\left|\beta_{j}\right|$ marks extrude above the shape $\lambda$ in the $j$ th column.

An $N$-semistandard (Young) tableau of shape $\lambda$ is an arrangement of numbers from $\{1,2, \ldots, N\}$ in the squares of $\lambda$ such that the entries strictly increase down each column and weakly increase across each row. A skew $N$ semistandard tableau of shape $\lambda / \theta$ is defined similarly, with entries placed in the squares of the skew shape $\lambda / \theta$. Let $(\lambda, \mu)$ be an $n$-marked $(n+m)$ shape. Then an $n$-marked $N$-semistandard tableau of shape $(\lambda, \mu)$ is just an $N$-semistandard tableau of shape $\lambda$ wherein the first $\beta_{j}$ squares, $n \geq \beta_{j} \geq 0$, in the $j$ th column are unmarked and the remaining $\alpha_{j}-\beta_{j}$ squares are marked. See Figure 1(b). Skew marked tableaux of shape $(\lambda / \theta, \mu)$ will also arise: The squares with entries will be exactly the squares of $\lambda / \theta$. Let $\delta_{j}$ be the $j$ th column length of $\theta$. The nonskew case can be considered skew by taking $\delta_{j}=0$ for $j \geq 1$. Sometimes it will happen that $\beta_{j}<\delta_{j}$. Then there will be $\delta_{j}-\beta_{j}$ marks in the $j$ th column where there are no entries.

We are now ready to define the central objects of this paper, viz. " $(n, m)$ symplectic tableaux". The definition is complicated. This paragraph and the following paragraph will most easily be read in conjunction with the first two paragraphs of $\S 3$, while consulting Figures 2-4. Let $N=2 n+m$. Throughout this paper we will work with $n$-marked $N$-semistandard tableaux with entries from the alphabet

$$
1<\overline{1}<2<\overline{2}<\cdots<n<\bar{n}<n+1<n+2<\cdots<n+m .
$$

The following technical artifice guarantees that there will always be at least one entry in any column which has marks in it. This artifice is not really needed for the tableaux which can actually arise from our algorithm, but it will make some proofs cleaner. Consult Figure 2 for the following two definitions. Given a tableau $T$ of $n$-marked shape $(\lambda / \theta, \mu)$, define the augmented tableau $\mathscr{A} T$ as follows: For $1 \leq j \leq \infty$, place the entry $\infty$ in the $\left(\alpha_{j}+1\right)$ st square of the $j$ th column, where $\alpha_{j}:=0$ for previously undefined column lengths $\alpha_{j}$ of $\lambda$. Mark the additional squares if they lie past the $n$th row or are below or to the right of an existing marked square. Given a marked tableau $T$ of shape $(\lambda, \mu)$ with entries from the alphabet above, define the $n$-skewing operation $\mathscr{S}_{n}$ as follows: Let $\theta$ be the region of squares in $\lambda$ which contain entries $\leq \bar{n}$. Remove these squares and their entries (but leave the marks alone), thereby creating the skew tableau $\mathscr{S}_{n} T$ of marked shape $(\lambda / \theta, \mu)$. Note that marks will extrude above $\lambda / \theta$ into the region $\theta$ if there were entries $\leq \bar{n}$ in marked 

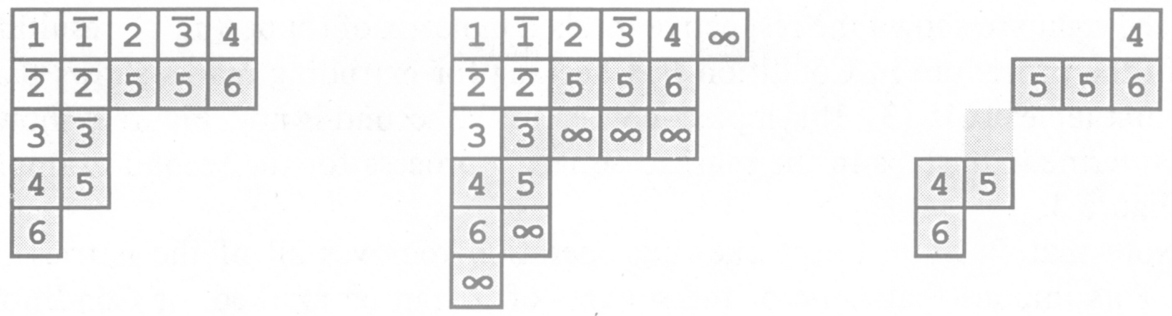

Figure 2. Tableaux $T, \mathscr{A} T$, and $\mathscr{S}_{n} T$ for $n=3$

squares. We will use the word squaring and the symbol $\mathscr{R}$ to refer to the jeu d'tacquin operation of converting a skew tableau to a nonskew tableau by sliding out empty squares. This operation will be defined at the beginning of $\S 3$ for both unmarked and marked skew tableaux.

Let $(\lambda, \mu)$ be an $n$-marked $(n+m)$-shape. An $(n, m)$-symplectic tableau of shape $(\lambda, \mu)$ is an $n$-marked $N$-semistandard tableau $T$ which satisfies the following two requirements:

Condition A. (" $n$-symplectic") If $i \leq n$, then the entries $i$ and $\vec{i}$ can occur no lower than the $i$ th row of $T$.

Condition B. ("marked jeu d'tacquin") If the augmenting, $n$-skewing, and marked squaring operations are applied to $T$, then no marked squares may extrude above the resulting nonskew tableau. In other words, if $\mathscr{R} \mathscr{S}_{n} \mathscr{A} T$ has $n$-marked shape $(\sigma, \tau)$, then $\tau$ must have nonnegative column lengths.

Condition A was invented by King when converting Zhelobenko's Gelfand patterns for $\mathrm{Sp}_{2 n}$ to semistandard form. A full appreciation of Condition B will be acquired while reading $\S \S 3-5$. For now, Figure 3 shows two $n$-marked $N$-semistandard tableaux which satisfy Condition A in the case $n=3$ and $m=3$.
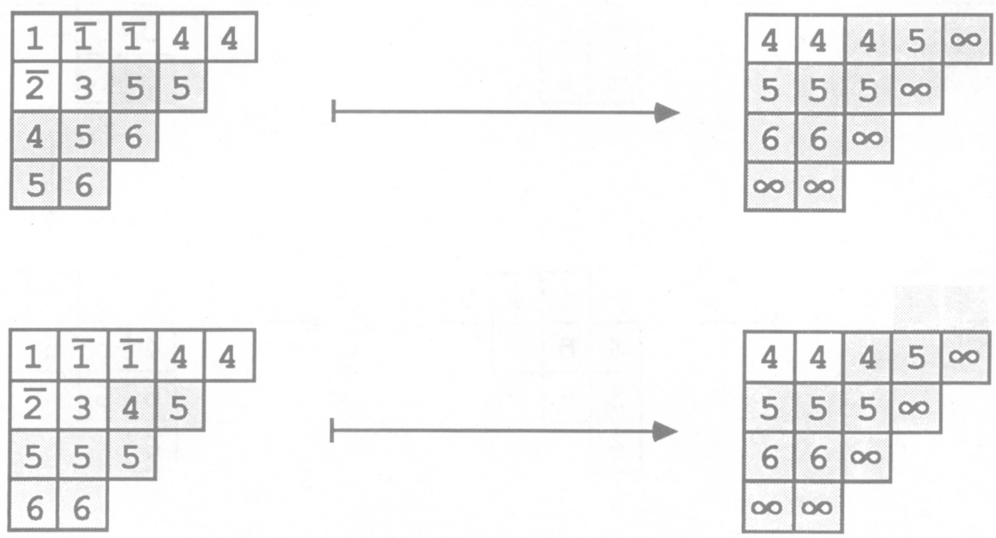

Figure 3. Tableaux $T, \mathscr{R}_{n} \mathscr{A} T, U$, and $\mathscr{R}_{n} \mathscr{A} U$ 
On the right are shown the respective marked tableaux of shape $(\sigma, \tau)$ resulting from the procedure in Condition B. Checking for extruding marks shows that the first tableaux is $(3,3)$-symplectic whereas the second is not. Figure 4 shows the intermediate steps in the marked squaring process for the second example in Figure 3.

Note that if $m=0$ the skewing operation removes all of the entries of $T$. This implies that none of the squares of $\lambda$ can be marked, or Condition B will be violated. Hence for the $\mathrm{Sp}_{2 n, 0}=\mathrm{Sp}_{2 n}$ case we must deal entirely with unmarked tableaux and only Condition A matters, which yields exactly the $2 n$-semistandard tableaux which Berele used. At the other extreme, if $n=0$ then there can be no entries of $T$ which are $\leq \bar{n}$, and so again Condition $\mathrm{B}$ is superfluous. And here Condition $\mathrm{A}$ is also superfluous, leaving us with the usual $m$-semistandard tableaux for $\mathrm{GL}_{m}=\mathrm{Sp}_{0, m}$. (Albeit with marks everywhere.) We will see below that Condition B is also superfluous in the case $\mathrm{Sp}_{2 n, 1}=\mathrm{Sp}_{2 n+1}$. So Condition $\mathrm{B}$ was difficult to discover because it is invisible in the known or recently solved cases of $\mathrm{GL}_{m}, \mathrm{Sp}_{2 n}$, and $\mathrm{Sp}_{2 n+1}$. In the general case, Condition A implies that entries $\leq \bar{n}$ must occur in the first $n$ rows. And as noted before, all squares in the $(n+1)$ st row and below are marked. Proposition 3.2 will show that all marked squares must have entries $\geq n+1$. The three conditions stated in the last three sentences rule out four of the following eight possibilities: (Square is marked or unmarked) $\times($ Square is in $\leq n$th or in $\geq(n+1)$ st row $) \times($ Entry is $\leq \bar{n}$ or $\geq n+1)$.

The odd symplectic groups occur when $m=1$, i.e. $N=2 n+1$. Then the definition of $(m, n)$-symplectic tableau could be made much simpler. There
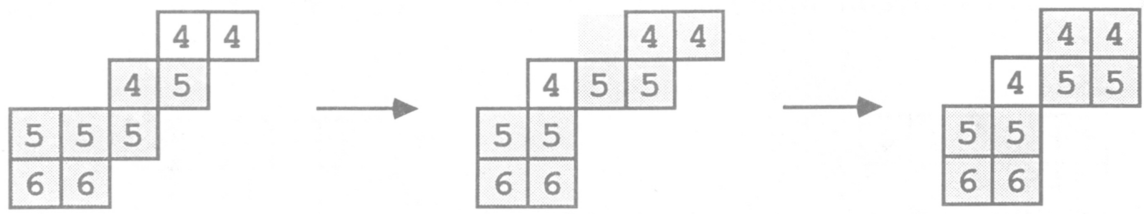

\begin{tabular}{|l|l|l|l|}
\hline 4 & 4 & 4 & 5 \\
\hline 5 & 5 & 5 & \multicolumn{1}{|c}{} \\
\cline { 1 - 2 } 6 & 6 & \multicolumn{2}{|c}{} \\
\cline { 1 - 2 } & &
\end{tabular}
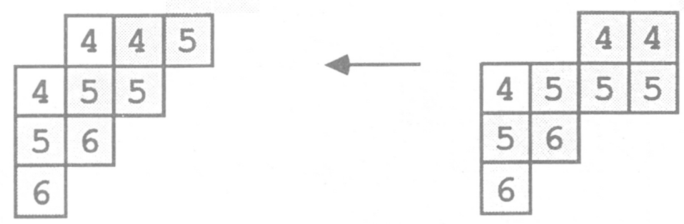

Figure 4. A marked skew tableau $T$, and after five sliding out operations, the squared marked tableau $\mathscr{R} T$ 
can be at most one marked square in each column, and some initial segment of consecutive columns will have all of the marked squares. So we would only need to keep track of the total number $d$ of marked squares instead of a region $\mu$; the shape would be denoted $(\lambda, d)$ instead of $(\lambda, \mu)$. And it will be seen in Proposition 3.2 that here each marked square must contain the entry $n+1$. Using the fact that the $n+1$ 's are the only entries $>\bar{n}$, it can easily be shown that Condition B will always be satisfied. So in addition to Condition A, one would only need to require that the last entry in each of the first $d$ columns is $n+1$.

Let $\lambda$ be an $m$-shape. The $\lambda$ th Schur function can be defined combinatorially by

$$
s_{\lambda}\left(z_{1}, \ldots, z_{m}\right)=\sum_{T} z_{1}^{\# 1 ' s(T)} \cdots z_{m}^{\# m s \mathbf{s}(T)},
$$

where the sum runs over all $m$-semistandard tableau $T$ of shape $\lambda$, and $\# i$ 's $(T)$ is the number of entries in $T$ equal to $i$. Let $\lambda$ be an $n$-shape. The symplectic analog to the $\mathrm{GL}_{m}$ Schur function $s_{\lambda}$ can be defined by using the KingZhelobenko description of the weights of the $\lambda$ th representation of $\mathrm{Sp}_{2 n}$ :

$$
\mathrm{sp}_{\lambda}\left(y_{1}, \ldots, y_{n}\right)=\sum_{T} y_{1}^{\# 1 \mathrm{~s}(T)-\# \overline{1} \mathbf{s}(T)} \cdots y_{n}^{\# n ' \mathrm{~s}(T)-\# \bar{n} \mathrm{~s}(T)},
$$

where the sum is over all $n$-symplectic $2 n$-semistandard tableaux $T$ of shape $\lambda$.

Let $(\lambda, \mu)$ be an $n$-marked $(n+m)$-shape. Motivated by the form of the output from our extension of Berele's algorithm, here we define intermediate symplectic Schur functions:

$$
\begin{aligned}
& \mathrm{sp}_{\lambda, \mu}\left(y_{1}, \ldots, y_{n} ; z_{1}, \ldots, z_{m}\right) \\
& \quad=\sum_{T} y_{1}^{\# 1 \mathrm{~s}(T)-\# \overline{1} \mathbf{s}(T)} \cdots y_{n}^{\# n^{\prime} \mathrm{s}(T)-\# \bar{n} \mathrm{~s}(T)} z_{1}^{\#(n+1) \mathrm{s}(T)} \cdots z_{m}^{\#(n+m)} \mathbf{s}(T),
\end{aligned}
$$

where the sum is over all $(n, m)$-symplectic tableaux $T$ of $n$-marked shape $(\lambda, \mu)$. By the remarks above concerning limiting cases, it is immediate that the $\mathrm{sp}_{\lambda, \mu}$ reduce to $s_{\lambda}$ when $n=0$ and to $\mathrm{sp}_{\lambda}$ when $m=0$.

Let $\lambda$ be a partition of $k$. Normally a standard Young tableau of shape $\lambda$ is thought of as an arrangement of the numbers $1,2, \ldots, k$ in the squares of the shape $\lambda$ such that the entries increase across each row and down each column. Here we will equivalently define an $m$-standard (Young) tableau of shape $\lambda$ to be a sequence of $k+1 m$-shapes

$$
\varnothing=\lambda^{(0)}, \lambda^{(1)}, \ldots, \lambda^{(i)}, \ldots, \lambda^{(k)}=\lambda,
$$

such that $\lambda^{(i+1)}$ is obtained from $\lambda^{(i)}$ by adjoining one square. Erase all of the numbers and all of the marks from Figure 7 to obtain an example of the following definition with $n=4$. An $n$-oscillating tableau of final shape $\lambda$ and length $k$ is a similarly notated sequence of $k+1 n$-shapes, except now $\lambda^{(i+1)}$ can also be obtained from $\lambda^{(i)}$ by removing a square. Erase only the numbers 
from Figure 7 for an example of the next definition. An $(n, m)$-oscillating tableau of final shape $(\lambda, \mu)$ and of length $k$ is a sequence of $k+1 n$-marked $(n+m)$-shapes

$$
(\varnothing, \varnothing)=\left(\lambda^{(0)}, \mu^{(0)}\right),\left(\lambda^{(1)}, \mu^{(1)}\right), \ldots,\left(\lambda^{(k)}, \mu^{(k)}\right)=(\lambda, \mu),
$$

such that $\lambda^{(i+1)}$ is obtained from $\lambda^{(i)}$ as before by adding or removing a square, and then $\mu^{(i+1)}$ is determined by $\mu^{(i)}, \lambda^{(i)}$, and $\lambda^{(i+1)}$ according to certain rules which are given in $\S 4$. Although the sequence of the $\lambda^{(i)}$ completely determines the $\mu^{(i)}$, it will be important to explicitly keep track of the $\mu^{(i)}$. Of particular importance is $\mu^{(k)}$, which describes the locations of the unmarked and marked squares in the final shape $\lambda^{(k)}$. The definition of $(n, m)$-oscillating tableau becomes much simpler when $n=1$, in a manner similar to that noted above for $(n, m)$-symplectic tableau.

Finally, $g_{\mu}(k, n)$ is defined to be the number of $n$-oscillating tableaux of final shape $\mu$ and length $k$. And $h_{\lambda, \mu}(k, n, m)$ is defined to be the number of $(n, m)$-marked oscillating tableaux of final marked shape $(\lambda, \mu)$ and length $k$.

\section{MARKed JeU D'TACQUiN}

Operations from the theory of jeu d'tacquin can be used to convert a skew semistandard tableau $T$ of shape $\lambda / \theta$ to a semistandard tableau of nonskew shape as follows. From the region $\theta$ of empty squares, choose a corner square, i.e. one which has two sides in common with the occupied region $\lambda / \theta$. Let $T_{r}$ be the entry of $T$ to the right and $T_{d}$ the entry below the chosen empty square. If $T_{r}<T_{d}$ then interchange the entry $T_{r}$ with the empty square; otherwise $T_{d} \leq T_{r}$ and then one interchanges the entry $T_{d}$ with the empty square. The empty square has moved one place to the right or down; repeat this process until the empty square "slides" out of the region $\lambda / \theta$ through the southeastern boundary. Then we have a skew semistandard tableau of shape $\lambda / \theta^{\prime}$ where $\left|\theta^{\prime}\right|=|\theta|-1$. Repeating the entire procedure many times eventually produces a semistandard tableau of nonskew shape. See Figure 4, ignoring the marks for now. It is a famous fact that the resulting tableau is independent of the order of choosing corner squares of the inner region [Tho].

Let $T$ be a skew semistandard tableau of $n$-marked shape $(\lambda / \theta, \mu)$. Let the "column lengths" $\beta_{j}$ of the unmarked region $\mu$ actually be integers (some possibly negative) such that $n \geq \beta_{1} \geq \beta_{2} \geq \cdots$. Convert $T$ into a tableau of ordinary shape as before. But now update the boundary of the region $\mu$ of unmarked squares after sliding out each empty square as follows: If the length of the $j$ th column of $\lambda / \theta$ decreases by 1 , then set $\beta_{j}^{\prime}=\beta_{j}-1$. And in order to keep the column lengths of $\mu$ a weakly decreasing sequence, also set $\beta_{h}^{\prime}=\beta_{h}-1$ for all $h \geq j$ such that $\beta_{h}=\beta_{j}$. When the auxiliary shape $\mu$ (i.e. the sequence of $\beta_{j}$ ) is included in the process, we will say that we are using marked jeu d'tacquin. With or without marks, we will say that the skew tableau 
of shape $\lambda / \theta$ has been squared when all of the squares from the empty region $\theta$ have been slid out. The resulting (marked) tableau is denoted $\mathscr{R} T$. When an arbitrary $n$-marked semistandard skew tableau is squared to a tableau of $n$-marked shape $(\lambda, \mu)$, it is possible for the $\beta_{j}$ sequence to become negative at some point. If $\beta_{j}<0$ then $\left|\beta_{j}\right|$ marks extrude above the diagram for $\lambda$ in the $j$ th column. Figure 4 illustrates the process of squaring a marked skew tableau.

Proposition 3.1. The process of squaring a marked skew tableau is well-defined; i.e., the resulting marked tableau is independent of the order of sliding out empty squares.

Proof. Consult Figure 5. Given a skew tableau of marked shape $(\lambda / \theta, \mu)$, remove the marks and then embed the tableau in a large skew semistandard tableau of shape $\rho / \theta$, where $\rho$ has column lengths $51+\beta_{j}$, as follows: In the first column adjoin the entries $100+\alpha_{1}+1,100+\alpha_{1}+2, \ldots, 150+\beta_{1}, \infty$; in the second column adjoin $200+\alpha_{2}+1,200+\alpha_{2}+2, \ldots, 250+\beta_{2}, \infty$; etc. Let $\chi$ be the region consisting of empty squares, single digit entries, and all three digit entries whose hundreds digits agrees with the column number, e.g. 208 in the second column. Initially the boundary of $\chi$ is just a translation by 50 rows of the original unmarked/marked boundary $\mu$, as illustrated by the heavy lines in Figure 5. As empty squares are slid out from $\theta$, we claim that the southeastern boundary of $\chi$ continues to model the unmarked/marked boundary. After the removal of an empty square, the leftmost column of $\chi$ which decreases (by
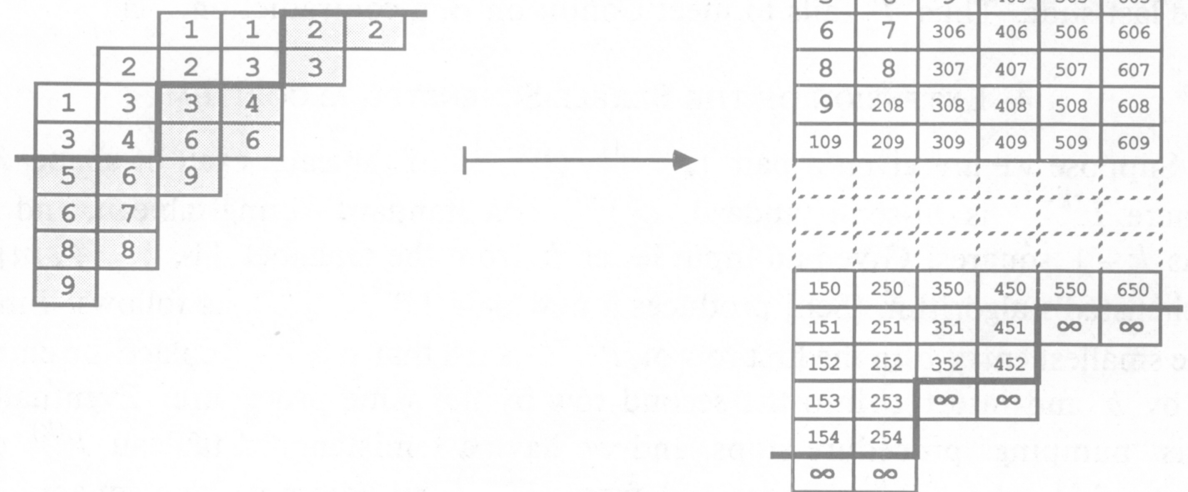

FIgURE 5. Proof of Proposition 3.1 
one) is the same as the leftmost column of $\lambda$ which decreases (by one). And if the $j$ th column of $\chi$ decreases, then all columns to the right of equal length will also decrease: The last entry in the $(j+1)$ st column of $\chi$ will be less than the first entry in the $j$ th column outside $\chi$, and similarly for the $(j+2)$ nd column, etc. In other words, empty squares always move along any horizontal portion of the $\chi$ boundary as they leave, thereby displacing three digit entries to the left of their original columns. So our claim is correct: The northwestern boundary of the region of all $\infty$ 's and displaced three digit numbers behaves like the northwestern boundary of the region of marks. Hence the final marked region is determined by the final locations of the adjoined entries. Apply the well definedness of ordinary jeu d'tacquin.

The following proposition helps to describe the nature of $(n, m)$-symplectic tableaux.

Proposition 3.2. All entries in marked squares of an $(n, m)$-symplectic tableau must be $\geq n+1$.

Proof. Let $T$ be an $(n, m)$-symplectic tableau of marked shape $(\lambda, \mu)$ such that the entries $\leq \bar{n}$ occupy a region $\theta \subseteq \lambda$. Suppose that $\theta \nsubseteq \mu$, in other words some marks in $\mathscr{S}_{n} \mathscr{A} T$ extrude into $\theta$. Consider the rightmost marked empty square of $\theta$. Columns to the left can be discarded. Slide out all empty squares from columns of $\theta$ which are to the right. At this point, at best, marks in columns to the right do not occur in rows above the mark in the $i$ th row that we are considering. All of the empty squares are in the first column; at best there are only $i$ of them. There is at least an $\infty$ in the first column of $\mathscr{S}_{n} \mathscr{A} T$ below the empty squares. All squares in rows $i$ and below are marked. Starting with the empty square in the $i$ th row, slide out the $i$ empty squares one at a time, noting the paths that they trace out. From the noncrossing of these paths one can deduce the standard jeu d'tacquin fact that the exit columns of the successively slid empty squares move left to right. Hence marks in the rightmost column move up once for each slide, resulting in an extrusion after the last slide. Thus $T$ fails to meet Condition B, a contradiction.

\section{Extension of the BeREle-Schensted ALGORITHM}

Suppose we are given a pair $\left(P^{(k-1)}, Q^{(k-1)}\right)$ of tableaux each of shape $\lambda$, where $P^{(k-1)}$ is $m$-semistandard, $Q^{(k-1)}$ is a standard Young tableau, and $\lambda$ has $k-1$ squares. Given an input letter $b$ from the alphabet $\{1,2, \ldots, m\}$, Schensted's algorithm [Sch] produces a new pair $\left(P^{(k)}, Q^{(k)}\right)$ as follows: Find the smallest entry $c$ in the first row of $P^{(k-1)}$ such that $c>b$. Replace the entry $c$ by $b$ and insert $c$ into the second row by the same procedure. Eventually this "bumping" procedure stops, and we have a semistandard tableau $P^{(k)}$ of shape $\lambda^{(k)}$ where $\lambda^{(k)}$ is obtained from $\lambda^{(k-1)}$ by adjoining one square. A standard tableau $Q^{(k)}$ is formed by taking $\mathbf{Q}^{(k-1)}$ and placing the entry $k$ in the newly adjoined square. Starting with $P^{(0)}=\varnothing$ and $Q^{(0)}=\varnothing$ and inputting 
a sequence of $k$ letters produces a pair $(P, Q)$ of the same shape. Given a pair $(P, Q)$ of the same shape, the process can be reversed to recover the input sequence. This yields the bijection codified by identity (1.1): Input the $m^{k}$ words of length $k$ to obtain all pairs $(P, Q)$ of the same shape $\lambda$ as $\lambda$ runs over all $m$-shapes with $k$ squares.

Berele's algorithm is an extension of Schensted's. Input a word of length $k$ from the alphabet $\{1, \overline{1}, 2, \overline{2}, \ldots, n, \bar{n}\}$. Proceed as before unless one finds an $i$ about to bump an $\bar{i}$ out of the $i$ th row, which would cause a violation of the $n$-symplectic condition. Then by definition the $i$ and the $\bar{i}$ annihilate each other, thereby creating an empty square at the former location of the $\bar{i}$. Slide this empty square out from $P$ to the southeast by using jeu d'tacquin. This produces an $n$-symplectic tableau $P^{(k)}$ of shape $\lambda^{(k)}$, where $\left|\lambda^{(k)}\right|=\left|\lambda^{(k-1)}\right|-1$. Now $Q$ is $n$-oscillating rather than $m$-standard: One simply keeps track of the history of shapes of $P^{(k)}$. Again the process can be reversed. Thus one obtains the bijection codified by identity (2): Input the $(2 n)^{k}$ words and obtain all pairs $(P, Q)$ of common shape $\lambda$ as $\lambda$ runs over all $n$-shapes with $k-2 l$ squares, where $l \geq 0$.

Since our intended inputs of words from $\{1, \overline{1}, \ldots, n, \bar{n}, n+1, \ldots$, $n+m\}$ are contained in the words from $\{1, \overline{1}, \ldots, n, \bar{n}, n+1, \overline{n+1}, \ldots$, $n+m, \overline{n+m}\}$, we can immediately apply Berele's algorithm. However it is not clear what the resulting possible outputs are. For an example let $n=1$ and $m=1$, and let $P^{(3)}$ and $Q^{(3)}$ be as in the top row of Figure 6. What was $P^{(2)}$ and the 3rd input? An annihilation must have occurred in either the first or second square of the first column. Berele's inverse procedure would in fact have a 2 annihilating a $\overline{2}$ in the second square. But here no $\overline{2}$ 's exist. And the annihilation cannot have occurred in the first square, for then the entry $\overline{1}$ would have been in the second row in $P^{(2)}$, which would have violated Condition A. So the given pair $\left(P^{(3)}, Q^{(3)}\right)$ cannot result from the input of a word in $\{1, \overline{1}, 2\}$. Such reasoning can always determine whether a given pair is in the output of Berele's algorithm applied to intermediate symplectic inputs. But the use of marks provides a more explicit description of the output.

Here is how we were led to the method of using marks. It is clear that the left tableau produced by Berele's algorithm must satisfy Condition A. Thus squares

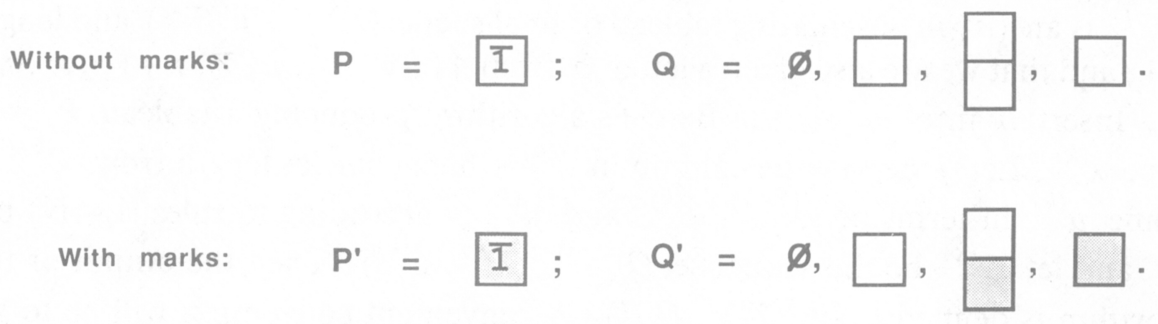

FIGURE 6. $P^{\prime}$ is not a possible output when $n=1, m=$ 1 , and $k=3$ 
in rows past the $n$th row must contain entries $\geq n+1$. We want these squares to continue to contain only entries $\geq n+1$ even if future annihilations raise the squares in the diagram. Hence we mark squares in $Q$ if they are created in the $m$ bottom-most rows (1). Subsequent annihilations make it possible for marked squares to appear in any row. Aesthetically and heuristically one is led to also postulate that the number of marks within a column never decreases (2), that the region of unmarked squares remains a legitimate shape in the upper left corner (3), and that squares should be marked only when necessary to satisfy the earlier requirements (4). (Representation theoretic experience helped to formulate the second postulate, which emphasizes the role of columns.) Rules (i)-(iv) below for defining $\mu^{(k)}$ in terms of $\mu^{(k-1)}, \lambda^{(k-1)}$, and $\lambda^{(k)}$ are completely determined by these four postulates. Alternatively, rules (i)-(iv) follow from partially formulating the intermediate Berele algorithm in terms of marked jeu d'tacquin, as stated in Proposition 4.1 below. (And if $m=1$, i.e., $N=2 n+1$, the polynomial methods of $\S 9$ provide a third way to arrive at Rules (i)-(iv).) In any case, a marked shape $(\lambda, \mu)$ which arises from applying (i)-(iv) to the $(n+m)$-oscillating tableau $\varnothing=\lambda^{(0)}, \lambda^{(1)}, \ldots, \lambda^{(k)}=\lambda$ can be easily characterized by (unmarked squares) $n \geq \beta_{1} \geq \beta_{2} \geq \cdots \geq 0$ and (marked squares) $m \geq \alpha_{1}-\beta_{1} \geq \alpha_{2}-\beta_{2} \geq \cdots \geq 0$ where $\beta_{j}$ and $\alpha_{j}=\beta_{j}+\left(\alpha_{j}-\beta_{j}\right)$ are the column lengths of $\mu$ and $\lambda$. Such a $(\lambda, \mu)$ is an $n$-marked $(n+m)$-shape.

The marked oscillating tableau $Q^{\prime}$ in the bottom row of Figure 6 shows the result of applying rules (i)-(iv) below to the original oscillating tableau $Q$. Our main result (Theorem 5.1) now immediately implies that $\left(P^{\prime}, Q^{\prime}\right)$ cannot possibly be an output when $n=1$ and $m=1$ because $P^{\prime}$ is not a $(1,1)$ symplectic tableau with respect to the final marked shape $(\lambda, \mu)$. It fails to satisfy Condition B.

We are now ready to describe our extension of Berele's algorithm to the intermediate symplectic cases. For an example, consult Figure 7, which shows the successive left tableaux $P^{(k)}$ resulting from inputting the letters of the given word from left to right.

Marked Berele Algorithm. Let $\left(\lambda^{(k-1)}, \mu^{(k-1)}\right)$ be an $n$-marked $(n+m)$-shape. Suppose that we are given a pair $\left(P^{(k-1)}, Q^{(k-1)}\right)$ where $P^{(k-1)}$ is an $N$ semistandard tableau of shape $\left(\lambda^{(k-1)}, \mu^{(k-1)}\right)$ satisfying Condition $\mathrm{A}$ and $Q^{(k-1)}$ is an $(n, m)$-oscillating tableau of final shape $\left(\lambda^{(k-1)}, \mu^{(k-1)}\right)$ and length $k-1$, and that we are also given a letter $b$ from $\{1, \overline{1}, \ldots, n, \bar{n}, n+1, \ldots, n+$ $m$ \}. Insert $b$ into $P^{(k-1)}$ via Berele's algorithm, producing a tableau $P^{(k)}$ of shape $\lambda^{(k)}$. Let $j$ denote the column in $\lambda^{(k)}$ which changes length from $\lambda^{(k-1)}$. Define $\mu^{(k)}$ in terms of $\lambda^{(k-1)}, \lambda^{(k)}$, and $\mu^{(k-1)}$ according to rules (i)-(iv) below, and let $Q^{(k)}$ be the sequence $Q^{(k-1)},\left(\lambda^{(k)}, \mu^{(k)}\right)$. Then the output of the algorithm is defined to be $\left(P^{(k)}, Q^{(k)}\right)$. A convenient convention will be to set $\alpha_{0}^{(h)}=n+m$ and $\beta_{0}^{(h)}=n$ for all $h \geq 0$ : By (ii) below this will force all squares in rows $n+1$ to $n+m$ to be marked. 

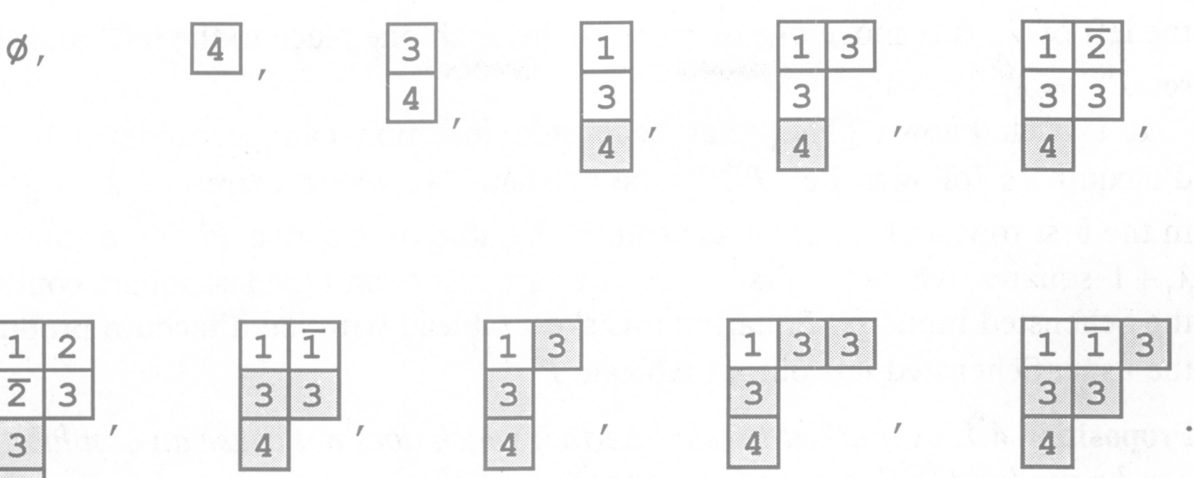

FIGURE 7. The successive left tableaux $P^{(k)}$ for $k=0$ to 10 for the input word $4313 \overline{2} 2 \overline{1} 13 \overline{1}$ where $n=2$ and $m=2$

(i) Adding a square-If possible insert an unmarked square and then slide down the marks in that column

$$
\alpha_{j}^{(k)}=\alpha_{j}^{(k-1)}+1, \quad \beta_{j}^{(k-1)}<\beta_{j-1}^{(k-1)} \Rightarrow \beta_{j}^{(k)}=\beta_{j}^{(k-1)}+1 .
$$

(ii) Adding a square-Otherwise add a marked square to the end of the column

$$
\alpha_{j}^{(k)}=\alpha_{j}^{(k-1)}+1, \quad \beta_{j}^{(k-1)}=\beta_{j-1}^{(k-1)} \Rightarrow \beta_{j}^{(k)}=\beta_{j}^{(k-1)} .
$$

(iii) Removing a square, easy case: Slide all of the existing marks in that column up one square

$$
\alpha_{j}^{(k)}=\alpha_{j}^{(k-1)}-1, \quad \beta_{j}^{(k-1)}>\beta_{j+1}^{(k-1)} \Rightarrow \beta_{j}^{(k)}=\beta_{j}^{(k-1)}-1 .
$$

(iv) Removing a square, hard case: Do as in (iii), and also mark all squares to the right of the raised marked square at $\left(\beta_{j}^{(k-1)}, j\right)$

and if

$$
\alpha_{j}^{(k)}=\alpha_{j}^{(k-1)}-1, \quad \beta_{j}^{(k-1)}=\beta_{j+1}^{(k-1)} \Rightarrow \beta_{j}^{(k)}=\beta_{j}^{(k-1)}-1,
$$

$$
\beta_{h}^{(k-1)}=\beta_{j}^{(k-1)} \text { for } h \geq j, \quad \beta_{h}^{(k)}=\beta_{h}^{(k-1)}-1 .
$$

The ten transitions in Figure 7 are of types (i), (i), (ii), (i), (i); (ii), (iv), (iii), (ii), and (i) respectively.

Although we will not use the following, it is interesting to note another version of this procedure. In terms of $m$-circled $(n+m)$-partitions, the parts of $\lambda^{(k)}$ are to be circled as follows: Suppose $\lambda^{(k)}$ is the same as $\lambda^{(k-1)}$ except $\lambda_{i}^{(k)}=\lambda_{i}^{(k)} \pm 1$. Simply circle "the same" (e.g. 2nd, 3rd, 5th, etc.) parts in $\lambda^{(k)}$ as in $\lambda^{(k-1)}$ except as follows. If $\lambda_{i}^{(k)}=\lambda_{i}^{(k-1)}+1=\lambda_{i-1}^{(k-1)}$ and $\lambda_{i}^{(k-1)}$ was uncircled but $\lambda_{i-1}^{(k-1)}$ was circled, then one or more circles should each be moved one place to the right to satisfy the equal parts convention. If $\lambda_{i}^{(k)}=\lambda_{i}^{(k-1)}-1$ and $\lambda_{i}^{(k-1)}$ was circled, then first move the circle from $\lambda_{i}$ to the rightmost uncircled $\lambda_{j}$ to 
the left of $\lambda_{i}$ (i.e. move one or more circles each one place to the left) and then set $\lambda_{i}^{(k)}=\lambda_{i}^{(k-1)}-1$.

It is well known [Tho] that Schensted insertion can be modeled by jeu d'tacquin as follows: Let $P^{(k-1)}$ be of shape $\lambda$, where there are $\lambda_{1}$ squares in the first row. Form a skew tableau $P^{\prime}$ by adjoining above $P^{(k-1)}$ a row with $\lambda_{1}+1$ squares, where the first $\lambda_{1}$ squares are empty and the last square contains the Schensted input $b$. Squaring this skew tableau with jeu d'tacquin produces the usual Schensted left output tableau $P^{(k)}$.

Proposition 4.1. A marked Berele insertion which does not cause an annihilation can be modeled with marked jeu d'tacquin in the same manner that Schensted insertion is modeled with ordinary jeu d'tacquin, i.e. rules (i) and (ii) above can be thought of as consequence of marked jeu d'tacquin. (However, in order to do this, on the left side of each left tableau we need to attach a dummy 0th column of length $m+n$ with entries $1,2, \ldots, n, n+1, \ldots, n+m$ wherein the last $m$ squares are marked.) If an annihilation does occur during a marked Berele insertion, then rules (iii) and (iv) above can be obtained by sliding out the resulting empty square with marked jeu d'tacquin.

Proof. Suppose that an insertion causes an annihilation. Perform the first part of the insertion with bumping until an annihilation occurs. The second assertion of this lemma is then just the trivial observation that (iii) and (iv) coincide with the marked jeu d'tacquin rules for adjusting marks after the empty square has been slid out. Now assume that no annihilation occurs. Then except for the marks we have ordinary Schensted insertion. Juxtapose the input $b$ to the northeast of $P^{(k-1)}$. Slide out the $\lambda_{1}$ empty squares from the first row, working from right to left. By the same standard jeu d'tacquin argument used in the proof of Proposition 3.2, the successive empty squares exit the bottoms of columns from right to left. Now one Schensted insertion causes exactly one column to increase by one square, and so in this context we know that exactly one column bottom stays in the same position while all of the others rise by one square. The right to left argument implies that within the rising columns the marked jeu d'tacquin phenomenon of adding marks to the right never occurs. Hence the marks end up where they started in these columns. However, the column with the stationary bottom will gain a mark as its length increases exactly when its original marked level was the same as the original marked level of the column to the left. So we thereby obtain rules (i) and (ii).

\section{MAIN RESUlt AND PROOF}

Theorem 5.1. The marked Berele algorithm specifies a bijection between the following two sets:

(I) All words of length $k$ from the alphabet $\{1, \overline{1}, 2, \overline{2}, \ldots, n, \bar{n}, n+$ $1, n+2, \ldots, n+m\}$. 
(II) The union over all $n$-marked $(n+m)$-shapes $(\lambda, \mu)$ (with $\lambda$ a partition of $k-2 l$ and $l \geq 0)$ of the sets of pairs $(P, Q)$ such that $P$ is an $(n, m)$-symplectic tableau of marked shape $(\lambda, \mu)$ and such that $Q$ is an $(n, m)$-oscillating tableau of length $k$ and final shape $(\lambda, \mu)$.

When stated in polynomial terms, this bijection becomes the identity (1.3).

When $n=1, m=2$, and $k=5$, all $n$-marked $(n+m)$-shapes $(\lambda, \mu)$ such that $\lambda$ is a partition of 5,3 , or 1 are shown in Figure 8 . The 2 nd, 4 th, 5th, 7th, 8th, 10th, and 12th marked shapes cannot occur as final shapes of $(m, n)$-oscillating tableaux when $k=5$. Hence there are 13 nonzero terms in the identity (1.3). Here we set $x_{i}=1$ and $y_{j}=1$ and list the terms $h_{\lambda, \mu}(5,1,2) \operatorname{sp}_{\lambda, \mu}(1 ; 1,1)$ in the same order as Figure 8:

$$
\begin{aligned}
(2 \times 1+2)^{5}= & 1024=1 \times 56+4 \times 64+5 \times 40+6 \times 16 \\
& +5 \times 8+4 \times 20+6 \times 4+11 \times 16 \\
& +5 \times 8+4 \times 2+9 \times 2+5 \times 4+5 \times 2 .
\end{aligned}
$$

The 5 th to last shape is the only one with $g_{\lambda, \mu}(5,1,2) \neq 0$ where Condition B comes into play. Here $\lambda=(2,1)$ and $\mu=(1)$. Condition A alone is satisfied by ten 4-semistandard tableaux for this $\lambda$, but two of these are eliminated by Condition B.

Proof of Theorem 5.1. By restricting from the $\{1, \overline{1}, \ldots, n, \bar{n}, n+1, \overline{n+1}$, $\ldots, n+m, \overline{n+m}\}^{k}$ context, from Berele's work we know that the algorithm without marks gives a bijection from $\{1, \overline{1}, \ldots, n, \bar{n}, n+1, \ldots, n+m\}^{k}$ to some set of pairs $\left(P^{(k)}, Q^{(k)}\right)$ where $P^{(k)}$ is $(2 n+m)$-semistandard and satisfies Condition A and where $Q^{(k)}$ is $(n, m)$-oscillating of length $k$. So we only need to confirm that the addition of marks to $P$ and $Q$ accurately describes the output image. Using induction on $k$, two things need to be checked. First, suppose that we are given $n$-marked $(n+m)$-shapes $\left(\lambda^{(k-1)}, \mu^{(k-1)}\right)$ and $\left(\lambda^{(k)}, \mu^{(k)}\right)$ which are the last two $n$-marked $(n+m)$-shapes of some $Q^{(k)}$ and that we are also given an $(n, m)$-symplectic tableau $P^{(k)}$ on $\left(\lambda^{(k)}, \mu^{(k)}\right)$. Then
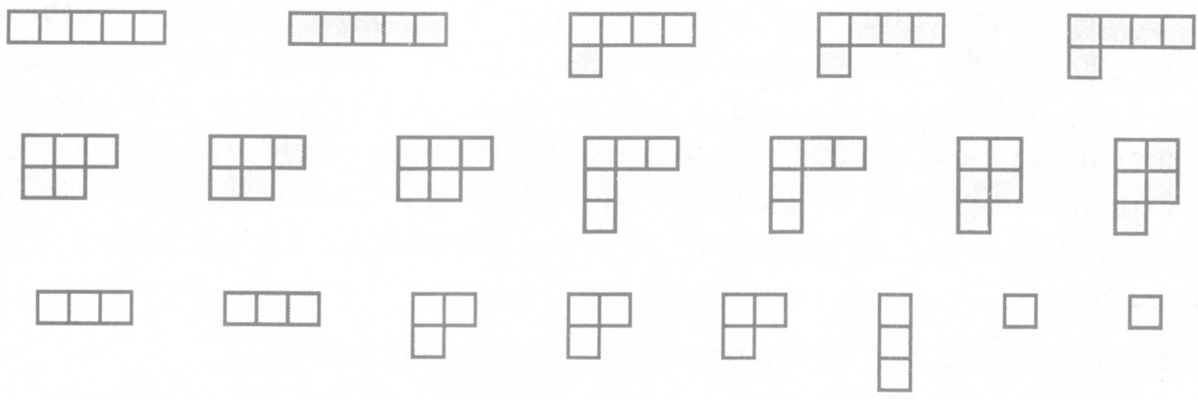

FIGURE 8. All 1-marked 3-shapes $(\lambda, \mu)$ such that $|\lambda|=$ 5,3 , or 1 
it must be checked that the $P^{(k-1)}$ constructed by the usual Berele inverse procedure contains only entries from $\{1, \overline{1}, \ldots, n, \bar{n}, n+1, \ldots, n+m\}$ : It is conceivable that an un-annihilation could occur which creates an $n+p$ and an $\overline{n+p}$ for some $p \geq 1$. Second, we must show that $P^{(k)}$ satisfies Condition $\mathrm{B}$ if and only if $P^{(k-1)}$ does. For the first point we need the following lemma.

Lemma 5.1. Suppose $P$ is $(n, m)$-symplectic on $(\lambda, \mu)$ and has entries $\leq \bar{n}$ in the nth row. Replace the rightmost such entry with an empty square and slide this empty square out. This empty square must exit at the bottom of a column which had $n$ unmarked squares to start with.

Proof. By Proposition 3.2 the original square at $(n, j)$ must be unmarked. Hence the $j$ th column has exactly $n$ unmarked squares. Suppose the square exits to the right from a column with fewer than $n$ unmarked squares. In such a column $j^{\prime}>j$ there is a marked square in the $n$th row. Let $q$ be the smallest $r$ such that the $r$ th row contains a marked square. So $q \leq n$. Begin to apply Condition B as follows. Starting with the $(n, j)$ entry and working from southwest to northeast, replace the last entry $\leq \bar{n}$ in each row by an empty square and slide the square out. After the first slide the marks in column $j^{\prime}$ move up. Hence $q^{(1)} \leq n-1$. Continuing as in the proof of Proposition 3.2, the remaining slides will exit farther to the northeast and further decrease the upper bound for $q$ by one at each step. So $q^{(n)} \leq 0$, i.e., there are extruding marks after $n$ slides.

Working on the first point in the proof of the theorem, assume that an annihilation occurred at the $k$ th step. Apply the inverse Berele procedure to $P^{(k)}$ : The unique square of $\lambda^{(k-1)}-\lambda^{(k)}$ is slid to the northwest from $(u, v)$ until moving it any further would violate Condition A. Call this punctured tableau $P^{(k)^{\prime}}$. Since Condition A was satisfied to start with, this implies that the empty square ends up at $(h, 1)$. If $h \leq n$ there is no problem with un-annihilating to an $h$ and an $\bar{h}$. Since only unmarked squares can be removed according to Rules (i)-(iv), the $v$ th column of $\lambda^{(k)}$ has $\leq n-1$ unmarked squares. Suppose $h \geq n+1$. Then the $n$th row of $P^{(k)^{\prime}}$ has at least one entry $\leq \bar{n}$. As in Lemma 5.1, replace the last entry $\leq \bar{n}$ in the $n$th row of $P^{(k)^{\prime}}$, at $(n, j)$, with an empty square. Slide the empty square at $(h, 1)$ back out to the southeast, re-producing $P^{(k)}$. This square exits at $(u, v)$. Now slide out the empty square from $(n, j)$. Since $n<h$ and $j \geq 1$, the usual jeu d'tacquin noncrossing path argument implies that this square exits to the northeast of $(u, v)$. Therefore it exits at the bottom of a column which had $<n$ unmarked squares. This contradicts Lemma 5.1 applied to $P^{(k)}$ and completes the confirmation of the first point.

For the second part of the proof, we need another lemma.

Lemma 5.2. The operations of skewing and squaring "commute" on skew marked tableaux which have been augmented, viz. $\mathscr{R} \mathscr{S}_{n} \mathscr{A} T=\mathscr{R} \mathscr{S}_{n} \mathscr{R} \mathscr{A} T$. 
Proof. (Augmenting $T$ first with the $\infty$ 's forces all marks to move correctly even after all entries $\leq \bar{n}$ have been removed by $\mathscr{S}_{n}$, thereby avoiding the minor pathology of having a column with marks but no entries.) Let $\mathscr{A} T$ be of skew marked shape $(\lambda / \theta, \mu)$, and let $\eta \supseteq \theta$ be the region consisting of all empty squares or squares with entries $\leq \bar{n}$. When computing $\mathscr{R} \mathscr{A} T$, note through which squares of $\eta$ successive empty squares from $\theta$ slide. The tableau $\mathscr{S}_{n} \mathscr{A} T$ has shape $(\lambda / \eta, \mu)$. When beginning to compute $\mathscr{R} \mathscr{S}_{n} \mathscr{A} T$, select the same sequence of squares from $\eta$ to slide out through $\mathscr{S}_{n} \mathscr{A} T$ and its successors. It is clear that finishing the computation of $\mathscr{R} \mathscr{S}_{n} \mathscr{A} T$ is the same as applying $\mathscr{R} \mathscr{S}_{n}$ to $\mathscr{R} \mathscr{A} T$.

Continuing the proof of the theorem, assume that no annihilation occurs when $b$ is inserted into $P^{(k-1)}$. By Proposition 4.1 we know that $P^{(k)}=$ $\mathscr{R}\left(P^{(k-1)} \cup b\right)$, where $\cup b$ means adjoining $b$ to the northeast. Since it is clear that the operations $\mathscr{A}$ and $\mathscr{R}$ commute on such slightly skew tableaux, we have $\mathscr{R} \mathscr{S}_{n} \mathscr{A} P^{(k)}=\mathscr{R} \mathscr{S}_{n} \mathscr{A} \mathscr{R}\left(P^{(k-1)} \cup b\right)=\mathscr{R} \mathscr{S}_{n} \mathscr{R} \mathscr{A}\left(P^{(k-1)} \cup b\right)$. So by Lemma 5.2 we have $\mathscr{R} \mathscr{S}_{n} \mathscr{A} P^{(k)}=\mathscr{R} \mathscr{S}_{n} \mathscr{A}\left(P^{(k-1)} \cup b\right)$. So extruding marks will occur in both or neither, i.e. $P^{(k)}$ satisfies Condition B if and only if $P^{(k-1)} \cup b$ does. If $b \leq \bar{n}$, then $\mathscr{S}_{n} \mathscr{A}\left(P^{(k-1)} \cup b\right)=\mathscr{S}_{n} \mathscr{A} P^{(k-1)}$. So $P^{(k-1)} \cup b$ satisfies Condition B if and only if $P^{(k-1)}$ does. If $b \geq n+1$, then first slide out the empty squares from $\mathscr{S}_{n} \mathscr{A}\left(P^{(k-1)} \cup b\right)$ which were formerly occupied by entries $\leq \bar{n}$ in $P^{(k-1)}$. Then it is clear that $\mathscr{R} \mathscr{S}_{n} \mathscr{A}\left(P^{(k-1)} \cup b\right)$ is just the Schensted insertion of $b$ into $\mathscr{R} \mathscr{S}_{n} \mathscr{A} P^{(k-1)}$. In general, given $U^{(k-1)}, U^{(k)}$, and $b$ such that $b$ and all entries of $U^{(k-1)}$ are $\geq n+1$ and such that $U^{(k)}$ is the result of inserting $b$ into $U^{(k-1)}$, then it is obvious that $U^{(k)}$ will have no extruding marks if and only if $U^{(k-1)}$ has none. Thus $\mathscr{R} \mathscr{S}_{n} \mathscr{A}\left(P^{(k-1)} \cup b\right)=\mathscr{R S}_{n} \mathscr{A} P^{(k)}$ has no extruding marks if and only if $\mathscr{R} \mathscr{S}_{n} \mathscr{A} P^{(k-1)}$ has none.

Now assume that an annihilation does occur when $b$ is inserted in $P^{(k-1)}$. Freeze the sliding out process when the newly created empty square is just about to leave the region in $P^{(k-1)}$ of squares occupied by entries $\leq \bar{n}$. Sliding out the empty square the rest of the way can be viewed as computing $P^{(k)}$ or as a first step in computing $\mathscr{R} \mathscr{S}_{n} \mathscr{A} P^{(k-1)}$. So applying the operation $\mathscr{R} \mathscr{S}_{n} \mathscr{A}$ to $P^{(k)}$ yields the same result as applying it to $P^{(k-1)}$. Hence in all cases we have shown that $P^{(k)}$ satisfies Condition B if and only if $P^{(k-1)}$ does.

\section{INTERMEDIATE SYMPLECTIC SCHUR FUNCTIONS AND MARKED SKEW SCHUR FUNCTIONS}

Here we obtain some basic properties of the intermediate symplectic Schur functions $\operatorname{sp}_{\lambda, \mu}\left(y_{1}, \ldots, y_{n} ; z_{1}, \ldots, z_{m}\right)$, which were defined near the end of $\S 2$. These are properties which one would expect tensor characters of $\operatorname{Sp}_{2 n, m}$ to have. Marked skew Schur functions are introduced.

Let the hyperoctahedral group $S_{n} \ltimes \mathbf{Z}_{2}^{n}$ act on $n$ variables and their inverses 
as follows: the variable $y_{i}$ can be interchanged with $y_{j}$ or with $y_{i}^{-1}$. Let the symmetric group $S_{m}$ act on the $m$ variables $z_{j}$ in the usual fashion.

Proposition 6.1. The intermediate symplectic Schur function $\operatorname{sp}_{\lambda, \mu}\left(y_{1}, \ldots, y_{n}\right.$; $\left.z_{1}, \ldots, z_{m}\right)$ is invariant under the action of the group $\left(S_{n} \times \mathbf{Z}_{2}^{n}\right) \times S_{m}$.

In order to prove the proposition we first need to define marked skew Schur functions. Recall [Mac] that the skew Schur function $s_{\lambda / \theta}\left(z_{1}, \ldots, z_{m}\right)$ can be combinatorially defined as the sum of weight monomials $z_{1}^{\# 1^{\prime}(T)} \cdots z_{m}^{\# m^{\prime} s(T)}$ corresponding to skew $m$-semistandard tableau $T$ of shape $\lambda / \theta$. Let $(\lambda / \theta, \mu)$ be a skew marked shape. The marked skew Schur function $s_{\lambda / \theta, \mu}\left(z_{1}, \ldots, z_{m}\right)$ is defined to be a sum of some of the same weight monomials as for $s_{\lambda / \theta}$. Now only weights of skew $m$-semistandard tableaux of marked shape $(\lambda / \theta, \mu)$ which satisfy Condition B of $\S 2$ are to enter in the sum: After augmenting and squaring, no marks can extrude above the resulting nonskew tableau. Note that by Proposition 3.2 we have $s_{\lambda / \theta, \mu}(z)=0$ if $\mu \nsucceq \theta$.

The proof of the following lemma is a variant of the main step in Schützenberger's proof of the Littlewood-Richardson rule, and in fact can be used to prove the rule [Pr2]. Our idea of enclosing everything in a rectangle, although a little artificial, seems slightly pedagogically simpler than Schützenberger's evacuation method. Let

$$
s_{\lambda / \theta}(z)=\sum_{\nu} c_{\lambda / \theta ; \nu} s_{\nu}(z)
$$

define the usual Littlewood-Richardson coefficients $c_{\lambda / \theta ; \nu}$.

Lemma 6.1. Marked skew Schur functions are symmetric in $z_{1}, \ldots, z_{m}$. In fact,

$$
s_{\lambda / \theta, \mu}(z)=\sum_{\nu} c_{\lambda / \theta, \mu ; \nu} s_{\nu}(z)
$$

where the coefficients $c_{\lambda / \theta, \mu ; \nu}$ are smaller integers than the original LittlewoodRichardson coefficients: $0 \leq c_{\lambda / \theta, \mu ; \nu} \leq c_{\lambda / \theta ; \nu}\left(=c_{\lambda / \theta, \lambda ; \nu}\right)$.

Proof. Refer to Figure 5 and the proof of Proposition 3.1. Given a skew $m$ semistandard tableau $T$ of marked shape $(\lambda / \theta, \mu)$, take the larger tableau constructed in the proof of Proposition 3.1 and embed it in a rectangular skew tableau with $50+\beta_{1}+1$ rows as follows: If $j>1$, adjoin $\infty+1, \infty+2, \ldots, \infty+$ $\beta_{j}-\beta_{1}$ to the bottom of the $j$ th column. Now slide out the empty squares from the empty region $\theta$. Using standard jeu d'tacquin arguments it is not hard to show that the shape of the resulting tableau is $\rho-\mu^{R}$ : Here the shape of the rectangle is denoted by $\rho$, whereas $\mu^{R}$ is the shape of $\mu$ rotated in $180^{\circ}$ in the plane, and - means to remove $\mu^{R}$ from the southeast corner of $\rho$. Let $\nu$ denote the (nonskew) shape of the region in the northwest corner now occupied by the original entries of $T$. Apply this process to all of the skew tableaux of shape $\lambda / \mu$ and collect some of the resulting nonskew $m$-semistandard tableaux $U$ : Discard $U$ if the $\chi$ boundary indicates that marks have extruded during 
the squaring process. In order to gather the outputs into Schur functions $s_{\nu}(z)$, we must show that any surviving $U$ of shape $\nu$ occurs as often as any other surviving $U^{\prime}$ of shape $\nu$. Let $C_{\lambda / \theta, \mu ; \nu}$ be the set of all tableaux (with entries $>100$ ) on the shape $\left(\rho-\mu^{R}\right) / \nu$ resulting from the process which have good $\chi$ boundaries. Concatenate any element $C$ of this set with any $m$-semistandard $U$ of shape $\nu$. Slide the empty squares in $\mu^{R}$ back up to the northwest corner. It is not hard to use standard jeu d'tacquin arguments to show that sliding all of the empty squares from $\mu^{R}$ to $\mu$ is the inverse of the original process of sliding all of the empty squares from $\mu$ to $\mu^{R}$. Hence any pair $(U, C)$ does come from some original $T$. Therefore any such $U$ occurs $c_{\lambda / \theta, \mu ; \nu}=\left|C_{\lambda / \theta, \mu ; \nu}\right|$ times.

Proof of Proposition 6.1. From the combinatorial definitions it is obvious that

$$
\mathrm{sp}_{\lambda, \mu}(y ; z)=\sum_{\theta \subseteq \lambda} \operatorname{sp}_{\theta, \theta}(y) s_{\lambda / \theta, \mu}(z) .
$$

But $\operatorname{sp}_{\theta, \theta}(y)$ is invariant under $S_{n} \ltimes \mathbf{Z}_{2}^{n}$ by Proposition 8.1 (or because it is a $\mathrm{Sp}_{2 n}$ character and the hyperoctahedral group is the Weyl group of $\mathrm{Sp}_{2 n}$ ). Applying Lemma 6.1 finishes the proof.

Lemma 6.2. The intermediate symplectic Schur functions $\operatorname{sp}_{\lambda, \mu}(y ; z)$ are linearly independent.

Proof. Given the $n$-marked $(m+n)$-shape $(\lambda, \mu)$, construct an $(n, m)$ symplectic tableau $T$ as follows: Place $i$ 's in the $i$ th row of $\mu$, where $1 \leq$ $i \leq n$. Place $n+i$ 's in the $i$ th marked square in each column. It is easy to see that $T$ satisfies both Conditions A and B. Define a total ordering of monomials: Monomials of greater degree precede monomials of smaller degree, and within degrees order lexicographically from the left with higher exponents coming earlier. For example,

$$
y_{1}^{7} y_{2}^{-3} y_{3}^{4} z_{1}^{2}<y_{1}^{7} y_{2}^{-3} y_{3}^{2} z_{1}^{4} \text {. }
$$

Then by Proposition 3.2 the tableau $T$ gives the first monomial of $\operatorname{sp}_{\lambda, \mu}(y ; z)$, and no other $\operatorname{sp}_{\lambda^{\prime}, \mu^{\prime}}(y ; z)$ has this monomial as its first monomial.

The following lemma comes from the crucial aspect of the main result of Sundaram's thesis [Sun].

Lemma 6.3. Apply the marked Berele algorithm to any two words in

$$
\{1, \overline{1}, \ldots, n, \bar{n}, n+1, \ldots, n+m\}^{k} .
$$

As in (II) of Theorem 5.1, denote the outputs $\left(P_{1}, Q_{1}\right)$ and $\left(P_{2}, Q_{2}\right)$. Now apply the ordinary Schensted algorithm to the same two words. Denote the outputs $\left(R_{1}, S_{1}\right)$ and $\left(R_{2}, S_{2}\right)$. If $Q_{1}=Q_{2}$, then $S_{1}=S_{2}$.

Proof. Exend the set of words to

$$
\{1, \overline{1}, \ldots, n, \bar{n}, n+1, \overline{n+1}, \ldots, n+m, \overline{n+m}\}^{k} .
$$


Apply the ordinary Berele algorithm for $\mathrm{Sp}_{2 n+2 m}$ to the same two words. Again $\left(P_{1}, Q_{1}\right)$ and $\left(P_{2}, Q_{2}\right)$ are output, but with no marks. But erasure of marks does not affect the equality $Q_{1}=Q_{2}$. And Schensted for $N=2 n+m$ embeds in Schensted for $N^{\prime}=2 n+2 m$. Now apply Theorem 10.8 of [Sun] in the $\mathrm{Sp}_{2 n+2 m}$ case.

If $\lambda$ is an $(n+m)$-shape, let $\lambda^{t r n c}$ be the $n$-shape consisting of the first $n$ rows of $\lambda$. We will call the $\operatorname{sp}_{\lambda, \mu}(y ; z)$ for which $\mu=\lambda^{\text {trnc }}$ trace-free intermediate symplectic Schur functions. (This terminology will be justified in Proposition 7.4.) The $n$-marked $(n+m)$-shapes $\left(\lambda, \lambda^{\text {trnc }}\right)$ have only the "mandatory" marks in the $(n+1)$ st to $(n+m)$ th rows of $\lambda$. These are exactly the $n$-marked shapes for which Condition B implies no additional restrictions upon the entries of an $(n, m)$-symplectic tableau beyond Condition A.

The decomposition of $s_{\eta}(x ; z)$ which is abstractly described in the following proposition is given explicitly for certain $\eta$ for arbitrary $n$ and $m$ in Proposition 7.2. Methods of computing the decomposition for arbitrary $\eta$ when $m=0$ are given in [K-T and $\mathrm{Ki} 2$ ].

Proposition 6.2. Upon specializing $x_{2 i-1}=y_{i}$ and $x_{2 i}=y_{i}^{-1}$, the Schur function $s_{\eta}\left(x_{1}, \ldots, x_{2 n} ; z_{1}, \ldots, z_{m}\right)$ decomposes uniquely into a nonnegative sum of intermediate symplectic Schur functions, i.e.,

$$
s_{\eta}(x \mapsto y ; z)=\sum_{(\lambda, \mu)} b_{\eta ; \lambda, \mu} \mathrm{sp}_{\lambda, \mu}(y ; z),
$$

where the $b_{\eta ; \lambda, \mu}$ are nonnegative integers. If $\eta$ is an $(n+m)$-shape, then the unique "leading term" is the trace-free $\operatorname{sp}_{\eta, \eta^{\text {trnc }}}(y ; z)$. (I.e. if $\eta$ has no more than $n+m$ rows and $|\eta|=k$, then $b_{\eta ; \eta, \eta^{\text {trnc }}}=1$ and $b_{\eta ; \lambda, \mu}=0$ for all other $\lambda$ such that $|\lambda|=k$.)

Proof. Suppose $|\eta|=k$. Use the ordinary Schensted algorithm to map

$$
\{1, \overline{1}, \ldots, n, \bar{n}, n+1, \ldots, n+m\}^{k} \text {, }
$$

to a union of pairs $(R, S)$ of tableaux. Fix a standard tableau $S_{0}$ of $(2 n+m)$ shape $\eta$. Let $W$ be the set of words of length $k$ which map to $\left(R, S_{0}\right)$, where $R$ can vary. Then the sum of the weight monomials corresponding to words in $W$ is $s_{\eta}\left(x_{1}, \ldots, x_{2 n} ; z_{1}, \ldots, z_{m}\right)$. Now apply the marked Berele algorithm to all of the words in $W$. Let $\left(P_{1}, Q_{1}\right)$ be an output of $n$-marked $n+m$-shape $(\lambda, \mu)$. Let $P_{2}$ be any $(n, m)$-symplectic tableau of shape $(\lambda, \mu)$. By Theorem 5.1 the pair $\left(P_{2}, Q_{1}\right)$ is a possible output. Lemma 6.3 implies that $\left(P_{2}, Q_{1}\right)$ is in the image of $W$. Hence the image of $W$ can be grouped into intermediate symplectic Schur functions $\operatorname{sp}_{\lambda, \mu}(y ; z)$. By Lemma 6.2 the decomposition is unique. If $\eta$ is an $(n+m)$-shape, there will be some words for which no annihilations occur. When the algorithm is restricted to such words it is just Schensted's algorithm. So the resulting tableaux will be of shape $\lambda=\eta$, and the only marks will be in rows $n+1$ and below. If any annihilations occur, then $|\lambda|<k$. 
There are many natural questions to ask regarding marked analogs of known constructions. Most of the results in Sundaram's thesis are candidates for generalization.

Problems. (1) Find a combinatorial interpretation of the "marked LittlewoodRichardson coefficients" $c_{\lambda / \theta, \mu ; \nu}$ i.e., what is the correct definition of the notion of "marked lattice permutation"?

(2) Generalize Sundaram's combinatorial description of the "branching" coefficients $b_{\eta, \lambda}$ for the case $m=0$ to the more general $b_{\eta ; \lambda, \mu}$ above for $m \geq 0$.

(3) Study all of this from the viewpoint of Knuth relations.

(4) (Problem 1 of [Sun, p. 143].) Given a $2 n$-semistandard tableau on the alphabet $\{1, \overline{1}, \ldots, n, \bar{n}\}$, show how to directly produce the corresponding $n$-symplectic tableau predicted by Sundaram's Theorem 10.8. Perhaps this can be done nicely from the $\mathrm{GL}_{2 n} \supset \mathrm{Sp}_{2,2 n-2} \supset \cdots \supset \mathrm{Sp}_{2 n-2,2} \supset \mathrm{Sp}_{2 n}$ marked Berele viewpoint. This would provide a combinatorial model for the restriction of the $\mathrm{GL}_{2 n}$ representation of type $\eta$ to the subgroup $\mathrm{Sp}_{2 n}$.

\section{TENSOR REPRESENTATIONS OF $\mathrm{Sp}_{2 n, m}$}

Let $V=\mathbf{C}^{2 n+m}$ be the space of column vectors upon which the intermediate symplectic group $\mathrm{Sp}_{2 n, m}$ acts from the left. The purpose of this section is to persuade the reader that the output of our marked Berele algorithm (i.e., (II) of Theorem 5.1) probably describes a nice decomposition of $\otimes^{k} V$ with respect to the action of $\mathrm{Sp}_{2 n, m}$. In particular, the characters of the pieces of the decomposition should be the intermediate symplectic Schur functions: Let $D_{2 n, m}(y ; z)$ denote a generic diagonal matrix in $\mathrm{Sp}_{2 n, m}$, viz. the diagonal matrix with entries $y_{1}, y_{1}^{-1}, \ldots, y_{n}, y_{n}^{-1} ; z_{1}, \ldots, z_{m}$. Let $\varphi$ be an appropriate piece of the representation of $\mathrm{Sp}_{2 n, m}$ on $\otimes^{k} V$. Then the character of $\varphi$, which by definition is $\operatorname{Trace}\left[\varphi\left(D_{2 n, m}(y ; z)\right)\right]$, should be some $\operatorname{sp}_{\lambda, \mu}(y ; z)$. In $\S 6$ it was shown that the $\operatorname{sp}_{\lambda, \mu}(y ; z)$ have some of the properties one would expect of tensor characters of $\mathrm{Sp}_{2 n, m}$.

Let a nonreductive group $G$ act on a vector space $\mathscr{V}$. A decomposition of $\mathscr{V}$ is a sequence of subspaces $0=\mathscr{W}_{0} \subseteq \mathscr{W}_{1} \subseteq \cdots \subseteq \mathscr{W}_{p}=\mathscr{V}$ such that the pieces $U_{i}=\mathscr{W}_{i} / \mathscr{W}_{i-1}$ are $G$ modules. A module $\mathscr{V}$ is indecomposable if $\mathscr{V} \neq \mathscr{U} \oplus \mathscr{W}$ for any proper $G$ submodules $\mathscr{U}$ and $\mathscr{W}$. The conjectured decompositions of the Young symmetry tensor spaces $\mathscr{V}^{\lambda}$ below will usually not be extreme in either direction. The $\mathscr{V}^{\lambda}$ will often be indecomposable to start with, but the conjecture will usually decompose $\mathscr{V}^{\lambda}$ somewhat on the one hand. On the other hand, the pieces of the decomposition will usually not be irreducible. Any representation $\varphi$ of $\mathrm{Sp}_{2 n, m}$ arising from a decomposition of $\otimes^{k} V$ is a polynomial representation: Given $g \in \mathrm{Sp}_{2 n, m}$, when a matrix for $\varphi(g)$ is written out with respect to some basis, then the entries of this matrix will be polynomials in the original matrix entries of $g$. The degree of 
an irreducible polynomial representation is the maximum degree of any matrix entry. Here we also define the degree of an arbitrary polynomial representation to be the minimum degree of an irreducible piece. A polynomial representation is isothermal if all of its irreducible pieces have the same degrees. For $l \geq 0$, let $\mathscr{D}_{2 l}^{k}$ be the subspace of $\otimes^{k} V$ obtained by taking the sum of all $\mathrm{Sp}_{2 n, m}$ submodules of degree at least $k-2 l$. We will give motivations for decomposing $\mathscr{V}^{\lambda}$ only part of the way and for the concept of degree later in this section.

Set $N=2 n+m$ and fix $k \geq 0$. Decompose $\otimes^{k} V$ into a direct sum of irreducible $\mathrm{GL}_{N}$ submodules $\mathscr{V}^{\eta}$ (each of which may occur more than once). Here $\eta$ is an $N$-partition and $\mathscr{V}^{\eta}$ is a subspace of $\otimes^{k} V$ of Young symmetry type $\eta$. Since $\mathrm{Sp}_{2 n, m} \subseteq \mathrm{GL}_{N}$, the subspace $\mathscr{V}^{\eta}$ is also an $\mathrm{Sp}_{2 n, m}$ module. Similarly, in the combinatorial context, Lemma 6.3 states that the Schensted and marked Berele algorithms are compatible. The original problem for this section was to relate all of the output (II) of Theorem 5.1 to a nice basis for all of $\otimes^{k} V$ with respect to the action of $\mathrm{Sp}_{2 n, m}$. But now by the compatibilities just noted, it can be seen that it suffices to focus attention on one $\mathscr{V}^{\eta}$ at a time. Let $D_{N}(x ; z)$ denote a generic diagonal matrix in $\mathrm{GL}_{N}$, viz. the diagonal matrix with entries $x_{1}, \ldots, x_{2 n} ; z_{1}, \ldots, z_{m}$. Then the Schur function $s_{\eta}\left(x_{1}, \ldots, x_{2 n} ; z_{1}, \ldots, z_{m}\right)$ is the character of $\mathrm{GL}_{N}$ acting on $\mathscr{V}^{\eta}$. When restricting from $\mathrm{GL}_{N}$ to $\mathrm{Sp}_{2 n, m}$, a generic torus element passes from $D_{N}(x ; z)$ to $D_{2 n, m}(y ; z)$. Hence Proposition 6.2 describes a decomposition of the $\mathrm{GL}_{N}$ character for $\mathscr{V}^{\eta}$ which conceivably could correspond to a decomposition of the representation with respect to $\mathrm{Sp}_{2 n, m}$. Restate Proposition 6.2 as follows:

$$
s_{\eta}(x \mapsto y ; z)=\sum_{(\lambda, \mu) \in B_{\eta}(n, m)} \operatorname{sp}_{\lambda, \mu}(y ; z),
$$

where $B_{\eta}(n, m)$ is a multiset which has $b_{\eta ; \lambda, \mu}$ copies of $(\lambda, \mu)$ in it.

We can now state the conjectured relationship between the marked Berele algorithm and representation theory. The main point of this conjecture is that $\otimes^{k} V$ should be decomposable into pieces whose characters are intermediate symplectic Schur functions, and that every such $\operatorname{sp}_{\lambda, \mu}(y ; z)$ can arise in this fashion.

Conjecture 7.1. Let $\eta$ be an $N$-partition of $k$. Consider an irreducible $\mathrm{GL}_{N}$ tensor module $\mathscr{V}^{\eta}$ of symmetry type $\eta$. Let

$$
\mathscr{V}_{2 l}^{\eta}=\left(\mathscr{V}^{\eta} \cap \mathscr{D}_{2 l}^{k}\right) /\left(\mathscr{V}^{\eta} \cap \mathscr{D}_{2 l-2}^{k}\right)
$$

which is a $\mathrm{Sp}_{2 n, m}$ module of degree $k-2 l$. There is a decomposition $\mathscr{W}_{1} \subseteq$ $\mathscr{W}_{2} \subseteq \cdots \subseteq \mathscr{W}_{p}$ of $\mathscr{V}_{2 l}^{\eta}$ such that the pieces $\mathscr{U}_{i}=\mathscr{W}_{i} / \mathscr{W}_{i-1}$ have the following properties: The $\mathscr{U}_{i}$ are isothermal indecomposable $\mathrm{Sp}_{2 n, m}$ modules of degree $k-2 l$. They are indexed by element $(\lambda, \mu)$ of the multiset $B_{\eta}(n, m)$ such that $|\lambda|=k-2 l$. The character of $\mathscr{U}_{i}$ is $\operatorname{sp}_{\lambda, \mu}(y ; z)$ if it is indexed by an element $(\lambda, \mu)$. There is a basis of weight vectors for $\mathscr{U}_{i}$ which are indexed 
by the $(n, m)$-symplectic tableaux of $n$-marked $(n+m)$-shape $(\lambda, \mu)$, and the $\mathrm{Sp}_{2 n, m}$ weight of a vector is equal to the weight of the indexing tableau.

If all of the irreducibles $\mathscr{V}^{\eta}$ occurring in a decomposition of $\otimes^{k} V$ are decomposed in this way, then the number of modules of $\mathrm{Sp}_{2 n, m}$ with characters $\operatorname{sp}_{\lambda, \mu}(y ; z)$ which occur overall is equal to the number of $(n, m)$-marked oscillating tableaux of length $k$ and final marked shape $(\lambda, \mu)$ by Theorem 5.1. Proposition 6.1 stated that the polynomials $\mathrm{sp}_{\lambda, \mu}(y ; z)$ are invariant under the action of $\left(S_{n} \times \mathbf{Z}_{2}^{n}\right) \times S_{m}$, which is the Weyl group of $\mathrm{Sp}_{2 n} \times \mathrm{GL}_{m}$. The parameters appearing in the conjecture are $n, m, \eta, k$, and $l$. As supporting evidence, we will now present confirmations for all reasonable extreme values for these parameters as well as for a couple of additional nonextreme small cases. Proofs are deferred until the end of the section. We start with the reductive cases, which have been known for years, and then consider extreme shapes. (The notation $x \mapsto y$ was defined in Proposition 6.2.)

Proposition 7.1. The conjecture is true for $n=0$ (i.e., for $\mathrm{GL}_{m} \subseteq \mathrm{GL}_{m}$ ) and for $m=0$ (i.e., for $\mathrm{Sp}_{2 n} \subseteq \mathrm{GL}_{2 n}$ ).

Proposition 7.2. The conjecture is always true for the following $\mathrm{GL}_{N}$ modules $\mathscr{V}^{\eta}$. If $\eta$ is a row of length $k$ (i.e., $\mathscr{V}^{\eta}=S^{k} V$ ), then the decomposition has one piece, viz.

$$
s_{\eta}(x \mapsto y ; z)=\operatorname{sp}_{\eta, \eta}(y ; z) .
$$

(Unless $n=0$ whence it is $\operatorname{sp}_{\eta, \phi}(y ; z)$.) If $\eta$ is an $N \times c$ rectangle (i.e., $\left.\mathscr{V}^{\eta}=S^{\mathcal{C}}\left[\bigwedge^{N} V\right]\right)$, then the decomposition has one piece, viz.

$$
s_{\eta}(x \mapsto y ; z)=\operatorname{sp}_{m \times c, \phi}(y ; z) .
$$

If $\eta$ is a column of length $k$ (i.e., $\mathscr{V}^{\eta}=\Lambda^{k} V$ ), then the decomposition is described by the identity

$$
s_{\eta}(x \mapsto y ; z)=\sum_{l=l_{b}}^{l_{f}} s_{\lambda, \mu}(y ; z) .
$$

Here $\lambda$ is a column of length $k-2 l$ and $\mu$ is a column of length $n-l$ unless $k-l<n$ whence the length of $\mu$ is $k-2 l$. And $l_{b}=0$ unless $k>m+n$ whence $l_{b}=k-n-m$; and $l_{f}=[k / 2]$ unless $[k / 2]>n$ whence $l_{f}=n$. If $\eta=(2,1)$, then the decompositions of $s_{\eta}(x \mapsto y ; z)$ for $n \geq 1$ and $m \geq 1$ are $\mathrm{sp}_{(2,1),(2)}(y ; z)+\mathrm{sp}_{(1),(1)}(y ; z)$ if $n=1$ and $\operatorname{sp}_{(2,1),(2,1)}(y ; z)+\operatorname{sp}_{(1),(1)}(y ; z)$ if $n \geq 2$.

When $k=3$ the only possibilities for $\eta$ are $(3),(2,1)$, and $(1,1,1)$. Hence Proposition 7.2 confirms the conjecture completely for $\otimes^{3} V$ for arbitrary $n$ and $m$. The smallest intermediate symplectic Schur function for which Condition B comes into play is $\operatorname{sp}_{(2,1),(1)}(y ; z)$ for $n=1$ and $m=2$. This polynomial first occurs as an output in Theorem 5.1 when $k=5$. And in fact 
(see the fifth case of the following proposition) $\operatorname{sp}_{(2,1),(1)}(y ; z)$ first occurs as an $\mathrm{sp}_{2 n, m}$ character for these same parameter values. This proposition indicates that Condition B does appear in a reasonable fashion in a representation theoretic context.

Proposition 7.3. The conjecture is true for all $\eta$ when $k=5, n=1$, and $m=2$. The decompositions are

$(3,1,1)$

$(2,2,1)$

$(2,1,1,1)$

$$
\frac{(\lambda, \mu)}{((5), \phi)}
$$

$$
\begin{gathered}
((1), \phi) ;((2,1),(2)) ;((3,2),(3)) \\
((2,1),(2)) ;((3), \phi) ;((3,1,1),(3))
\end{gathered}
$$

$((1), \phi) ;((1,1,1),(1)) ;((2,1),(1)) ;((2,2,1),(2))$

$((1,1,1),(1)) ;((2,1), \phi)$

In each case bases can be chosen so that each representation "feeds into" the sum of the representations listed to its right.

Finally, Proposition 7.4 below states that we know that the case $l=0$ (i.e. degree $k$ ) of the conjecture is always true. The $l=0$ piece of $s_{\eta}(x \mapsto y ; z)$ was described in Proposition 6.2. The representations corresponding to $l=0$ are also especially notable. Let $e_{1}, e_{\overline{1}}, \ldots, e_{n}, e_{\bar{n}}, e_{n+1}, \ldots, e_{n+m}$ and $f_{1}, f_{\overline{1}}, \ldots, f_{n}, f_{\bar{n}}, f_{n+1}, \ldots, f_{n+m}$ denote the axis basis vectors for $V$ and $V^{*}$. Then $f_{i}\left(e_{j}\right)=\delta_{i j}$. In this notation,

$$
B_{2 n, m}=\sum_{i=1}^{n}\left(f_{i} \otimes f_{\bar{i}}-f_{\bar{i}} \otimes f_{i}\right)=: B .
$$

A rank $k$ tensor $T \in \bigotimes^{k} V$ can be described by the $N \times N \times \cdots \times N$ ( $k$ times) array $T^{i_{1} \cdots i_{k}}$, where

$$
T=\sum_{i_{k}=1}^{N} \cdots \sum_{i_{1}=1}^{N} T^{i_{1} \cdots i_{k}} e_{i_{1}} \otimes \cdots \otimes e_{i_{k}} .
$$

The $(p, q)$ th contraction of $T$ is defined to be the rank $k-2$ tensor with components

$$
\sum_{i_{q}=1}^{N} \sum_{i_{p}=1}^{N} B_{i_{p} i_{q}} T^{i_{1} \cdots i_{p} \cdots i_{q} \cdots i_{k}},
$$

where $B_{i j}$ is the component matrix of $B$. The tensor $T$ is said to be tracefree if all of its $\left(\begin{array}{l}k \\ 2\end{array}\right)$ contractions are zero. For any subspace $\mathscr{V} \subseteq \bigotimes^{k} V$, let $\mathscr{V}_{0}$ denote the subspace of trace free tensors in $\mathscr{V}$. By Lemma 7.2 below the two definitions of the symbol $\mathscr{V}_{0}^{\eta}$ made so far agree. Weyl realized the irreducible finite dimensional representations of $G=\mathrm{O}_{N}$ or $\mathrm{Sp}_{2 n}$ by showing that the $\mathscr{V}_{0}^{\eta}$ for appropriate $\eta$ were irreducible $G$ modules. In [Pr1] we show that if $\eta$ is an $(n+m)$-shape, then $\mathscr{V}_{0}^{\eta}$ is an indecomposable $\operatorname{Sp}_{2 n, m}$ 
module which has a weight basis indexed by the semistandard tableaux of shape $\eta$ which satisfy only Condition A. Recall that Condition B is vacuous for $n$ marked shapes $\left(\eta, \eta^{t r n c}\right)$. So the character of $\mathscr{V}_{0}^{\eta}$ is indeed the "trace-free" function $\operatorname{Sp}_{\eta, \eta^{t r n c}}(y ; z)$. Now if $\eta$ has more than $n+m$ rows, it is easy to use combinatorial reasoning as in $\S 6$ to see that there are no $\operatorname{sp}_{\lambda, \mu}(y ; z)$ with $|\lambda|=k$ appearing in $s_{\eta}(x \mapsto y ; z)$. By first restricting from $\mathrm{GL}_{N}$ to $\mathrm{GL}_{2 n}$ as in Equation I.5.9 of [Mac], and then from $\mathrm{GL}_{2 n}$ to $\mathrm{Sp}_{2 n}$ as in [K-T], one can see that $\mathscr{V}^{\eta} \cap \mathscr{D}_{0}^{k}=0$ if $\eta$ has more than $n+m$ rows. We have confirmed the trace-free cases of the conjecture:

Proposition 7.4. The conjecture is always true when $l=0$ : If $\eta$ has more than $n+m$ rows then $\mathscr{V}^{\eta} \cap \mathscr{D}_{0}^{k}=\{0\}$ and no $\operatorname{sp}_{\lambda, \mu}(y ; z)$ with $|\lambda|=k$ appear in the decomposition of $s_{\eta}(x \mapsto y ; z)$. Otherwise, if $\eta$ is an $(n+m)$ partition, $\mathscr{V}^{\eta} \cap \mathscr{D}_{0}^{k}=\mathscr{V}_{0}^{\eta}$ is the subspace of trace-free tensors in $\mathscr{V}^{\eta}$. This is an indecomposable $\mathrm{Sp}_{2 n, m}$ module with character $\operatorname{sp}_{\eta, \eta^{\text {trnc }}}(y ; z)$, which is the unique $\operatorname{sp}_{\lambda, \mu}(y ; z)$ appearing in $s_{\eta}(x \mapsto y ; z)$ with $|\lambda|=k$.

Here are some comments on the problem of decomposing $\otimes^{k} V$ with respect to the action of $\mathrm{Sp}_{2 n, m}$. Even in the $m=0$ case of $\mathrm{Sp}_{2 n}$ the problem of explicitly specifying a basis for each irreducible piece of $\mathscr{V}^{\eta}$ is still open. (The $\mathrm{Sp}_{2 n}$ analog of Young symmetrizing projection operators is not known; however see $[\mathrm{R}-\mathrm{W}]$.) Berele [Be2] has constructed a basis for $\mathscr{V}_{0}^{\eta}$ realized as a quotient of $\mathscr{V}^{n}$. But the $\mathrm{Sp}_{2 n}$ case does permit an abstract approach to decomposing $\otimes^{k} V$ via the theory of characters of semisimple groups. By the classification of finite dimensional irreducible representations of semisimple Lie groups, all possible characters for $\mathrm{Sp}_{2 n}$ are known à priori. Furthermore, representations are determined by characters. Hence when $m=0$, the restriction of $\mathscr{V}_{\lambda}$ from $\mathrm{GL}_{2 n}$ to $\mathrm{Sp}_{2 n}$ is reduced to a polynomial calculation.

Unlike the situation for $\mathrm{Sp}_{2 n}$, where it is known that every tensor representation is equivalent to a trace-free tensor representation, a complete set of appropriate tensor characters are not known à priori for $\mathrm{Sp}_{2 n, m}$. In addition, characters no longer determine representations uniquely. (E.g. the $\mathrm{Sp}_{2 n+1}$ modules $V /\left\langle\mathbf{C} e_{n+1}\right\rangle \oplus \bigwedge^{2 n+1} V$ and $V$ both have character $y_{1}+y_{1}^{-1}+\cdots+y_{n}+y_{n}^{-1}+z$.) What is meant by appropriate? One could consider decompositions into irreducible representations. Then one would really be considering $\mathrm{Sp}_{2 n} \times \mathrm{GL}_{m}$ representations. Let $\nu$ denote the defining ("natural") representation of $\mathrm{Sp}_{2 n, m}$ on $V$. When $m=1$, the representation $\nu$ decomposes into irreducible representations of dimensions $2 n$ and 1 . The point of studying "trace-free" representations in $[\mathrm{Pr} 1]$ was to explain the existence of gap-filling identities with respect to characters of $\mathrm{SO}_{2 n}, \mathrm{SO}_{2 n+1}$, and $\mathrm{Sp}_{2 n}$. The foremost example of this is that the dimension of $\nu$, viz. $2 n+1$, fills a gap between $2 n$ and $2 n+2$. So we do not want to decompose $\nu$. On the other hand, there are some entire $\mathscr{V}_{\lambda}$ upon which the action of $\mathrm{Sp}_{2 n, m}$ is indecomposable. We are seeking an 
intermediate (not minimal or maximal) module decomposition

$$
\mathscr{V}_{0}^{\lambda} \subseteq \mathscr{V}_{1}^{\lambda} \subseteq \cdots \subseteq \mathscr{V}_{f}^{\lambda}=\mathscr{V}^{\lambda}
$$

such that each $\mathscr{V}_{i}^{\lambda} / \mathscr{V}_{i-1}^{\lambda}$ is an indecomposable $\mathrm{Sp}_{2 n, m}$ module with some $\mathrm{sp}_{\lambda, \mu}(y ; z)$ as character.

Motivation for choosing a particular decomposition of $\otimes^{k} V$ from the many decompositions possible is provided by the goal of keeping track of the copies of (roughly speaking) the bilinear form which defines $\mathrm{Sp}_{2 n, m}$. (For $\mathrm{Sp}_{2 n}$, the bilinear form $B_{2 n, m}$ is nondegenerate and provides an identification of $V^{*} \otimes V^{*}$ with $V \otimes V$. Then $B_{2 n, m}$ itself can be considered as an element of $V \otimes V$.) In $V \otimes V$ define

$$
A_{2 n, m}:=\sum_{i=1}^{n}\left(e_{i} \otimes e_{\bar{i}}-e_{\bar{i}} \otimes e_{i}\right)=: A .
$$

Note that the $(1,2)$-contraction of $A$ is the scalar $2 n$. Set $\mathscr{T}^{k}=\bigotimes^{k} V$. Note that

$$
\mathscr{T}^{2}=\mathscr{T}_{0}^{2} \oplus \mathbf{C} A,
$$

in other words $A$ provides a complementary notion to that of "trace-free". Here $\oplus$ means vector space direct sum. In fact the sum is direct with respect to $\mathrm{Sp}_{2 n} \times \mathrm{GL}_{m}$ but not $\mathrm{Sp}_{2 n, m}$. Using this fact $\left(\begin{array}{l}k \\ 2\end{array}\right)$ times and intersecting, one obtains

$$
\mathscr{T}^{k}=\mathscr{T}_{0}^{k} \oplus A \otimes \otimes \mathscr{T}^{k-2},
$$

where $\otimes \otimes$ means to tensor multiply in all possible positions. Let

$$
\mathscr{T}_{2 s}^{k}=A \otimes \otimes A \otimes \otimes \cdots \otimes \otimes A \otimes \otimes \mathscr{T}_{0}^{k-2 s},
$$

where there are $s$ factors of $A$. Iterating the above result, we get

Lemma 7.1. $\mathscr{T}^{k}=\bigoplus_{s=0}^{[k / 2]} \mathscr{T}_{2 s}^{k}$.

A major goal of Conjecture 7.1 is to reconcile this decomposition of $\mathscr{T}^{k}$ with the decomposition of $\mathscr{T}^{k}$ into Young symmetry pieces:

$$
\mathscr{T}^{k}=\bigoplus \mathscr{V}^{\lambda}
$$

where the sum is over a multiset. For an example, note that

$$
\mathscr{T}^{2}=V \otimes V=S^{2} V \oplus \bigwedge^{2} V
$$

is the Young symmetry decomposition for rank 2 tensors. Since $A \in \Lambda^{2} V$, it is easy to combine the two decompositions:

$$
\mathscr{T}^{2}=S^{2} V \oplus\left[\bigwedge^{2} V\right]_{0} \oplus \mathbf{C} A
$$

When $m=0$ the second sum is direct. Otherwise the third piece "feeds into" the second piece, which is indecomposable (but not irreducible). Let $\mathscr{C}_{2 l}^{k}=$ $\bigoplus_{j=0}^{l} \mathscr{T}_{2 j}^{k}$. We omit the proof of the following lemma. 
Lemma 7.2. $\mathscr{D}_{2 l}^{k}=\mathscr{C}_{2 l}^{k}$ and hence $\mathscr{D}_{2 l}^{k} / \mathscr{D}_{2 l-2}^{k}=\mathscr{C}_{2 l}^{k} / \mathscr{C}_{2 l-2}^{k} \cong \mathscr{T}_{2 l}^{k}$.

This lemma implies that the conjectured $\mathrm{Sp}_{2 n, m}$ representations of degree $k-2 l$ "contain" $l$ copies of $A$. Since $A \in \Lambda^{2} V$, it is reasonable to picture $A$ as a verticle $2 \times 1$ domino. Note that Berele's algorithm can be slightly reformulated as follows: At the usual annihilation step, instead let the entry $i$ bump the entry $\bar{i}$ out of the $(i, 1)$ square into square $(i+1,1)$. Now erase both entries, thereby creating an empty vertical domino. Sliding the two empty squares out gives the original result. In the case $m=0$, when $\eta$ is an $n$-partition, it is known [Lit, p. 295] that

$$
s_{\eta}(x \mapsto y)=\sum c_{\eta / \theta ; \mu} \mathbf{s p}_{\mu}(y),
$$

where the sum is over all $\theta \subseteq \eta$ with all column lengths even, and $c_{\eta / \theta ; \mu}$ is a Littlewood-Richardson coefficient as defined in $\S 6$. Heuristically this can be interpreted as follows. Suppose that $|\eta|=k$. Given an irreducible $\mathrm{GL}_{2 n}$ representation $\mathscr{V}^{\eta}$, pave a subshape $\theta \subseteq \eta$ with $l$ vertical dominos corresponding to copies of $A$. Skew tableaux on the remaining skew shape $\eta / \theta$ somehow describe a sum of trace free tensor representations of rank $k-2 l$. Perhaps rigorous versions of these heuristic ideas could be used to prove Conjecture 7.1 or to solve Problem (4) of $\S 6$.

Proof of Proposition 7.1. If $n=0$ then $\mathscr{V}^{\eta} \cap \mathscr{D}_{0}^{k}=\mathscr{V}^{\eta}$ and $\mathscr{V}^{\eta}$ does not decompose at all; here $s_{\eta}(x \mapsto y ; z)=\mathrm{sp}_{\eta, \phi}(y ; z)=s_{\eta}(z)$. If $m=0$ then $\mathrm{Sp}_{2 n, m}=\mathrm{Sp}_{2 n}$ is semisimple and the decomposition of $\mathscr{V}^{\eta}$ is determined by the decomposition of $s_{\eta}(x \mapsto y)$ into $\operatorname{sp}_{\lambda}(y)$ 's (which is described for all $2 n$ partitions $\eta$ in [K-T]). The decomposition respects degrees. The existence of weight bases in both cases is well known.

Proof of Proposition 7.2. If $\mathscr{V}^{\eta}=S^{k} V$, then it is easy to see that $\mathscr{V}^{\eta}=$ $\left[\mathscr{V}^{\eta}\right]_{0}$. Then apply Proposition 7.4. At the opposite extreme, suppose that $\mathscr{V}^{\eta}=S^{c}\left[\bigwedge^{N} V\right]$. This is a one-dimensional representation with character [det $g]^{c}=\left(z_{1} z_{2} \cdots z_{m}\right)^{c}$. So it has degree $m c$, and in fact $s_{N \times c}(x \mapsto y ; z)=$ $\mathrm{sp}_{m \times c, \phi}(y ; z)$.

Now suppose that $\mathscr{V}^{\eta}=\Lambda^{k} V$. The decomposition of $s_{\eta}(x \mapsto y ; z)$ can be found using algorithmic reasoning. Since $\eta$ is a column of length $k$, the words mapping to $s_{\eta}(x ; z)$ under Schensted are strictly decreasing sequences of length $k$. Apply the marked Berele algorithm to these words. We claim that $Q^{(j)}$ is always a column, and that as $j=0$ to $k$, the length of $Q^{(j)}$ increases up to some $j=j_{0}$ and then decreases thereafter: Suppose the earliest annihilation, viz. $i$ with $\bar{i}$ in the $i$ th row, occurs at step $j_{0}+1$. Then the next insertion would shove something $<i$ into the $i$ th row if no annihilation occurs. So once annihilations start to occur, they keep happening. Conversely, suppose that an input word is not strictly decreasing. Let $b$ be the first nondecreasing letter. If $b$ follows a 1 annihilating a $\overline{1}$, then $b$ either causes a reincrease in the 
column length or it goes into the second column. In all other cases $b$ just goes into the second column. So the converse is true. Let $Q$ be such a sequence of columns, ending with $Q^{(k)}$ of length $\alpha$. Let $l=k-j_{0}$ be the number of annihilations. Then $\beta=k-2 l-\left(j_{0}-n\right)=n-l$ squares are unmarked, unless the column never dips into the marked region, whence $k-2 l$ squares are unmarked. Let $\lambda$ and $\mu$ be columns of lengths $\alpha$ and $\beta$ respectively, so that $Q^{(k)}=(\lambda, \mu)$. By Theorem 5.1 , every tableau for $\operatorname{sp}_{\lambda, \mu}(y ; z)$ corresponding to $Q$ comes from some word of length $k$. But we have shown that right tableaux of this form arise exactly from strictly decreasing words. So $s_{\eta}(x \mapsto y ; z)$ is the sum of $\operatorname{sp}_{\lambda, \mu}(y ; z)$ over all such possible $Q$. The final length $k-2 l$ must be nonnegative, at no time can the length exceed $n+m$, and no more than $n$ annihilations can occur. So $l_{b} \leq l \leq l_{f}$ for $l_{b}$ and $l_{f}$ as stated.

The decomposition of $s_{\eta}(x \mapsto y ; z)$ in the one column case can also be computed explicitly with polynomial computations involving elementary symmetric functions. We omit this proof. The confirmation of the vector space decomposition into indecomposables with weight bases asserted in Conjecture 7.1 is accomplished by following through the polynomial proof with vector spaces and observing how the unipotent part of the Lie algebra of $\mathrm{Sp}_{2 n, m}$ acts.

If $\eta=(2,1)$ then it is easy to use Young symmetrizing operators to confirm the conjecture for the cases $n=1$ and $n \geq 2$.

Proof of Proposition 7.3. Young symmetrizing operators were used to give bases for each $\mathscr{V}^{\eta}$. Most weights for these $\mathrm{GL}_{4}$ representations have multiplicity one. Occasionally we had to take appropriate linear combinations in the multiplicity two and three cases. Then $\mathrm{Sp}_{2 n, m}$ invariance and indecomposability were checked by hand.

\section{Determinantal EXPRESSIONS}

The goal of this section and the next is to provide polynomial proofs of the $m=0$ (or $\mathrm{Sp}_{2 n}$ ) and $m=1$ (or $\mathrm{Sp}_{2 n+1}$ ) cases of the identity (1.3). A similar proof for the $n=0$ (or $\mathrm{GL}_{m}$ ) case can be easily constructed by following the proofs given here. Nothing more than sophisticated high school algebra will be used. We believe that the methods and viewpoints used here may be useful elsewhere, e.g. for the orthogonal groups. Therefore each step will be done as generally as possible to make clearer just what the essential part is.

As usual, set $N=2 n+m$. In this section, $x$ will sometimes denote $x_{1}, \ldots, x_{N}$ instead of $x_{1}, \ldots, x_{2 n}$. Define the polynomials $e_{k}\left(x_{1}, \ldots, x_{N}\right)$ and $f_{k}\left(x_{1}, \ldots, x_{N}\right)$ as follows:

$$
e_{k}\left(x_{1}, \ldots, x_{N}\right):=\sum_{1 \leq i_{1}<\cdots<i_{k} \leq N} x_{i_{1}} \cdots x_{i_{k}}
$$

and

$$
f_{k}\left(x_{1}, \ldots, x_{N}\right):=e_{k}\left(x_{1}, \ldots, x_{N}\right)-e_{k-2}\left(x_{1}, \ldots, x_{N}\right) .
$$


Given a sequence of integers $\alpha_{1}, \ldots, \alpha_{c}$, use a $c \times c$ determinant to define

$$
M_{c}(\alpha ; f(x))=\frac{1}{2}\left|f_{\alpha_{j}-j+i}(x)+f_{\alpha_{j}-j+2-i}(x)\right|_{1 \leq i, j \leq c} .
$$

This is sometimes called a "formal character". In the course of computation, determinants of undesired forms can arise. These are dealt with by using "modification rules" (as in e.g. [Ki2]). The first of these states that $M_{c}(\alpha ; f)$ is dependent only on the shape $\lambda$ with column lengths $\alpha_{j}$ (i.e., is unaffected by extra trailing 0's). (The notation $x \mapsto y$ was defined in Proposition 6.2.)

Lemma 8.1. (M1) If $\alpha_{c}=0$, then $M_{c}(\alpha ; f)=M_{c-1}\left(\alpha^{\prime} ; f\right)$, where $\alpha^{\prime}$ is the sequence $\alpha_{1}, \ldots, \alpha_{c-1}$.

(M2) If $\alpha=n+1$ and $N=2 n$ then $M_{c}(\alpha ; f(x \mapsto y))=0$.

Proof. Rule (M1) is obvious. For rule (M2), first note that $e_{k}(x \mapsto y)=$ $e_{2 n-k}(x \mapsto y)$. Then $f_{n+i}(x \mapsto y)=-f_{n+2-i}(x \mapsto y)$ and the first column vanishes.

Lemma 8.2. Suppose $\alpha_{1} \geq \alpha_{2} \geq \cdots \geq \alpha_{c}>0$. Then

$$
M_{c}\left(\alpha ; f\left(x_{1}, \ldots, x_{N}\right)\right)=\sum x_{N}^{d} M_{c^{\prime}}\left(\alpha^{\prime} ; f\left(x_{1}, \ldots, x_{N-1}\right)\right),
$$

where the sum is over all $\alpha_{1}^{\prime} \geq \alpha_{2}^{\prime} \geq \cdots \geq \alpha_{c^{\prime}}^{\prime}>0$ where $\alpha_{j}^{\prime}=\alpha_{j}$ or $\alpha_{j}-1$ (omit all $\alpha_{j}^{\prime}=0$ ), and where $d$ is the number of $\alpha_{j}^{\prime}=\alpha_{j}-1$.

Proof. Apply $f_{k}\left(x_{1}, \ldots, x_{N}\right)=x_{N} f_{k-1}\left(x_{1}, \ldots, x_{N-1}\right)+f_{k}\left(x_{1}, \ldots, x_{N-1}\right)$ to every term in $M_{c}(\alpha ; f(x))$. Split each column in two and expand to get a sum of $2^{c}$ determinants $M_{c}\left(\alpha^{\prime} ; f\right)$. Having two identical columns will cause any $M_{c^{\prime}}\left(\alpha^{\prime} ; f\right)$ with $\alpha_{j}^{\prime}=\alpha_{j+1}^{\prime}-1$ to vanish. And use (M1) if any trailing $\alpha_{j}^{\prime}=0$.

Recall that $\operatorname{sp}_{\lambda, \lambda^{\text {trnc }}}(y ; z)$, the trace-free intermediate symplectic Schur functions, were defined in $\S 7$ before Proposition 7.4. The following Giambelli-type determinantal expression for trace-free $\mathrm{sp}_{\lambda, \mu}$ with $n+1$ or fewer rows generalizes Equation 3.4 of [Ki2]. All symplectic Schur functions for the case $m=0$ and all trace-free intermediate symplectic Schur functions for the case $m=1$ are among the cases covered by this proposition. See Proposition 9.1 for arbitrary $\mathrm{sp}_{\lambda, \mu}$ in the $m=1$ case. Somewhat different determinantal expressions for trace-free $\mathrm{sp}_{\lambda, \mu}$ with any number of rows can be written down using [G-V].

Proposition 8.1. Let $\lambda$ be an $(n+1)$-shape with column lengths $\alpha_{1} \geq \alpha_{2} \geq \cdots \geq$ $\alpha_{c}>0$. Then

$$
\operatorname{sp}_{\lambda, \lambda^{r r c c}}(y ; z)=M_{c}\left(\alpha ; f\left(x_{2 i-1} \mapsto y_{i}, x_{2 i} \mapsto y_{i}^{-1}, x_{2 n+j} \mapsto z_{j}\right)\right) .
$$

Proof. In each $(n, m)$-symplectic tableau of shape $\left(\lambda, \lambda^{\text {trnc }}\right)$, temporarily replace $y_{1}, y_{1}^{-1}, \ldots, y_{n}, y_{n}^{-1}, z_{1}, \ldots, z_{m}$ by $x_{1}, x_{2}, \ldots, x_{2 n-1}, x_{2 n}, x_{2 n+1}$, $\ldots, x_{2 n+m}$ respectively. Then by inspection of their definition the $\operatorname{sp}_{\lambda, \lambda^{t r n c}}(x)$ satisfy the recursion of Lemma 8.2 in the order $x_{N}, x_{N-1}, \ldots, x_{1}$, except 
with the added condition on the sum of $\alpha_{1}^{\prime} \leq[N / 2]$. Compare initial conditions. Both $M_{0}(\phi ; f(x))=1$ and $\operatorname{sp}_{\phi}(x)=1$ for zero or more variables. And if $\alpha_{1}=n+1$ and $N=2 n$, then both $\operatorname{sp}_{\lambda, \lambda^{r r n c}}\left(y_{1}, \ldots, y_{n}\right)=0$ and $M_{c}\left(\alpha ; f\left(y_{1}, y_{1}^{-1}, \ldots, y_{n}, y_{n}^{-1}\right)\right)=0$ by combinatorial inspection and rule (M2) respectively. So $\mathrm{sp}_{\lambda, \lambda^{\text {trnc }}}$ and $M_{c}(\alpha ; f(y))$ satisfy exactly the same recursion. The initial conditions determine the recursion solutions for all $\lambda$ with no more than $n+1$ rows. (If $\alpha_{1}=n+2$ then $M_{c}\left(\alpha ; f\left(y_{1}, \ldots, y_{n}\right)\right.$ ) $\neq 0$ in general but $\operatorname{sp}_{\lambda, \lambda^{\text {trnc }}}(y)=0$.)

Corollary 8.1. If $N=2 n$ and $\lambda$ is a column of length $k \leq n$ then $\operatorname{sp}_{\lambda, \lambda}(y)=$ $f_{k}(y)$.

\section{Polynomial proOfS FOR $\mathrm{Sp}_{2 n}$ AND $\mathrm{Sp}_{2 n+1}$}

When $m=0$ or $m=1$ the following theorem together with induction on $k$ can be used instead of Theorem 5.1 to prove the identity (1.3).

Theorem 9.1. (a) Let $\lambda$ be an n-shape. Then

$$
\left(y_{1}+y_{1}^{-1}+\cdots+y_{n}+y_{n}^{-1}\right) \operatorname{sp}_{\lambda, \lambda}(y)=\sum \operatorname{sp}_{\lambda^{\prime}, \lambda^{\prime}}(y) \text {, }
$$

where the sum is over all $n$-shapes $\lambda^{\prime}$ which can be obtained from the shape $\lambda$ by adjoining or removing one square.

(b) Let $(\lambda, \mu)$ be an n-marked $(n+1)$-shape. Then

$$
\left(y_{1}+y_{1}^{-1}+\cdots+y_{n}+y_{n}^{-1}+z\right) \operatorname{sp}_{\lambda, \mu}(y ; z)=\sum \operatorname{sp}_{\lambda^{\prime}, \mu^{\prime}}(y ; z),
$$

where the sum is over all $n$-marked $(n+1)$-shapes $\left(\lambda^{\prime}, \mu^{\prime}\right)$ such that $\lambda^{\prime}$ can be obtained from $\lambda$ by adjoining or removing a square, and then $\mu^{\prime}$ is determined by $\lambda, \mu$, and $\lambda^{\prime}$ according to the rules (i)-(iv) of $\S 4$.

When $m=0$ let $\operatorname{sp}_{\mu}(y):=\operatorname{sp}_{\mu, \mu}(y)$. Suppose $m=1$. Consider an $n$ marked $(n+1)$-shape $(\lambda, \mu)$ : If $|\lambda / \mu|=b$ then $(\lambda, \mu)$ must consist of $\mu$ together with an additional marked square at the end of each of the first $b$ columns. Then set $(\mu, b):=(\lambda, \mu)$ and $\operatorname{sp}_{\mu, b}(y ; z):=\operatorname{sp}_{\lambda, \mu}(y ; z)$. Throughout this section the column lengths of $\mu$ are $\beta_{1} \geq \beta_{2} \geq \cdots \geq \beta_{c} \geq 0$ where $c \geq b \geq 0$. The following lemma is an immediate consequence of the combinatorial definitions and Proposition 3.2.

Lemma 9.1 ( $\mathrm{Sp}_{2 n+1} \downarrow \mathrm{Sp}_{2 n}$ branching).

$$
\operatorname{sp}_{\mu, b}\left(y_{1}, \ldots, y_{n} ; z\right)=\sum_{\theta} z^{t} \operatorname{sp}_{\theta}\left(y_{1}, \ldots, y_{n}\right),
$$

where the sum runs over all n-shapes $\theta$ with column lengths $\delta_{j^{\prime}}=\beta_{j^{\prime}}$ for $1 \leq j^{\prime} \leq b$ and $\delta_{j}=\beta_{j}$ or $\beta_{j}-1$ for $j>b$ and where $t=b+d$, with $d$ being the number of decremented columns.

Now define "formal characters" for arbitrary $n$-marked $(n+1)$-shapes when $N=2 n+1$. Here $\beta_{1}, \ldots, \beta_{c}$ are arbitrary integers and $c \geq b \geq 0$. Let 


$$
\begin{aligned}
& z f_{k}(x)=z f_{k}\left(x_{1}, \ldots, x_{2 n}\right) \text { and } f_{k}(x, z)=f_{k}\left(x_{1}, \ldots, x_{2 n}, z\right) \text {. Define } \\
& N_{b, c}(\beta ; z f(x), f(x, z)) \\
& \quad:=\frac{1}{2}\left|z f_{\beta_{j}-j+i}(x)+z f_{\beta_{j}-j+2-i}(x) \vdots f_{\beta_{j}-j^{\prime}+i}(x, z)+f_{\beta_{j}-j^{\prime}+2-i}(x, z)\right|,
\end{aligned}
$$

where the rows are indexed by $1 \leq i \leq c$, and the two kinds of columns are indexed by $1 \leq j \leq b<j^{\prime} \leq c$. The proof of the following proposition is similar to the proof of Proposition 8.1 via Lemma 8.2. But now only the $x_{2 n+1}=z$ to $x_{2 n}$ recursion given in Lemma 9.1 is needed, with Proposition 8.1 providing the initial conditions at $N=2 n$.

Proposition 9.1. Let $(\mu, b)$ be an $n$-marked $(n+1)$-shape. Then

$$
\operatorname{sp}_{\mu, b}(y ; z)=N_{b, c}(\beta ; z f(x \mapsto y), f(x \mapsto y, z)) .
$$

The main step in the proof of Theorem 9.1, viz. Lemma 9.2 below, is a determinant identity which can be stated in a very general setting. For example, with appropriate modification rules, it could also be used as the main step for an orthogonal analog of Proposition 9.1. Consider a collection of variables $t_{k j}$ where $j \geq 1$ and $k \in \mathbf{Z}$. For any sequence of integers $\gamma_{1}, \ldots, \gamma_{c}$, define a polynomial $L_{c}(\gamma ; t)$ by:

$$
L_{c}(\gamma ; t)=\left|t_{\gamma_{j}-j+i, j}+t_{\gamma_{j}-j+2-i, j}\right|_{1 \leq i, j \leq c} .
$$

Note that the variables $t_{k j}$ appear exactly in the $j$ th column. For the following lemma take the $(c+1)$ st set of variables to be $t_{k, c+1}=0$ for $k<0$, and $t_{0, c+1}=1$ and $t_{1, c+1}=u$; the higher values of $k$ are irrelevant. And define $\varepsilon_{j}=\left(0_{1}, 0_{2}, \ldots, 1_{j}, \ldots, 0_{c \text { or } c+1}\right)$, so that if $\gamma^{\prime}=\gamma+\varepsilon_{i}$, then $\gamma_{i}^{\prime}=\gamma_{i}+1$ and $\gamma_{j}^{\prime}=\gamma_{j}$ otherwise.

Lemma 9.2 (General Pieri multiplication by one box).

$$
u L_{c}(\gamma ; t)=L_{c+1}\left(\gamma+\varepsilon_{c+1} ; t\right)+\sum_{j=1}^{c} L_{c}\left(\gamma+\varepsilon_{j} ; t\right)+\sum_{j=1}^{c} L_{c}\left(\gamma-\varepsilon_{j} ; t\right) .
$$

Proof. For the sake of clarity take $c=3$ and $\gamma_{1}=p, \gamma_{2}=q, \gamma_{3}=r$. Drop the kernel $t$ when displaying determinants, i.e., write $p+1, p-1$ for $t_{p+1,1}+t_{p-1,1}$. Expanding over the last column,

$$
L_{c+1}\left(\gamma+\varepsilon_{c+1} ; t\right)=u L_{c}(\gamma ; t)-D_{c}(\gamma ; t) \text {, }
$$

where

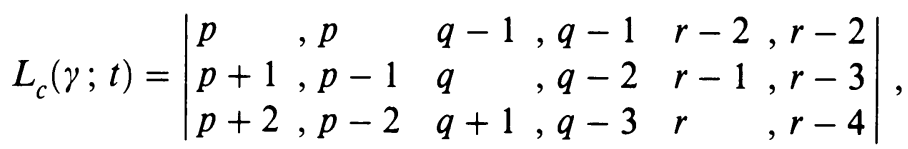

and

$$
D_{c}(\gamma ; t)=\left|\begin{array}{llll}
p & , p & q-1, q-1 & r-2, r-2 \\
p+1, p-1 & q & q-2 & r-1, r-3 \\
p+3, p-3 & q+2, q-4 & r+1, r-5
\end{array}\right| \text {. }
$$


Write the six terms of the sums $\sum_{j=1}^{c} L_{c}\left(\gamma+\varepsilon_{j} ; t\right)$ and $\sum_{j=1}^{c} L_{c}\left(\gamma-\varepsilon_{j} ; t\right)$ out:

$$
\begin{aligned}
& \left|\begin{array}{lllll}
p+1, p+1 & q-1 & , q-1 & r-2 & , r-2 \\
p+2, p & q & , q-2 & r-1 & , r-3 \\
p+3, p-1 & q+1, q-3 & r & , r-4
\end{array}\right|
\end{aligned}
$$

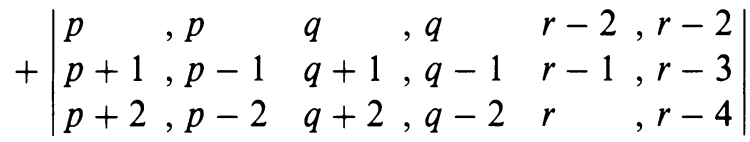

$$
\begin{aligned}
& +\cdots+\left|\begin{array}{llll}
p \quad, p & q-1, & q-1 & r-3, r-3 \\
p+1, p-1 & q & , q-2 & r-2, r-4 \\
p+2, p-2 & q+1, & q-3 & r-1, r-5
\end{array}\right| .
\end{aligned}
$$

Within each binomial color the first term red and the second term blue. When compared to the matrix for $L_{c}(\gamma ; t)$, any term from one of the six determinants has exactly one "displaced" factor. Using colors to help keep track of things, analysis of signs and displaced factors shows that almost all of the terms from the six determinants cancel (usually respecting colors). We omit a 15 sentence argument which is not completely obvious. The sum of the surviving terms is $D_{c}(\gamma ; t)$; focusing on the bottom row of this determinant helps one to recognize the surviving terms in the sum of the six determinants.

For the proof of Theorem 9.1 we will be working at four different levels. The determinants $L_{c}(\gamma ; t)$ in the variables $t_{i j}$ are very general. At the next level (of formal characters) we have the $M_{c}(\gamma ; f(x))$ or $N_{b, c}(\gamma ; z f(x), f(x, z))$ in the sets of variables $t_{k j}=f_{k}(x)$ or $z f_{k}(x)$ and $f_{k}(x, z)$. These sets of variables vanish for negative indices, e.g. $f_{-1}=0$. At this level we do not assume that the $\gamma_{j}$ are weakly decreasing or that $\gamma_{j} \leq n$. At the third level we work with the quantities $M_{c}\left(\beta ; f\left(y, y^{-1}\right)\right)$ and $N_{b, c}\left(\beta ; z f\left(y, y^{-1}\right), f\left(y, y^{-1}, z\right)\right)$. In addition to specializing $x \mapsto y$, we assume that the $\beta_{j}$ form a weakly decreasing sequence. The fourth level consists of the combinatorially defined $\operatorname{sp}_{\mu}\left(y_{1}, \ldots, y_{n}\right)$ and $\operatorname{sp}_{\mu, b}\left(y_{1}, \ldots, y_{n} ; z\right)$. By Propositions 8.1 and 9.1 the third and fourth levels coincide, provided that $\mu$ has no more than $n$ rows. In addition to Lemma 8.1 we will need the following modification rules. The trivial rule $(\mathrm{GM})$ has already been used in the proofs of Lemma 8.2 and Proposition 9.1 .

\section{Lemma 9.3.}

(GM) If the $j$ th and $(j+1)$ st columns use the same variables, viz. $t_{k, j}=t_{k, j+1}$, then $L_{c}(\gamma ; t)=0$ if $\gamma_{j+1}=\gamma_{j}+1$.

(N0) If $\beta_{b}=\beta_{b+1}-1=\cdots=\beta_{b+a}-1$ for some $a \geq 1$, then $N_{b, c}(\beta ; z f, f)=$ $N_{b+a, c}\left(\beta^{\prime} ; z f, f\right)$ where $\beta_{b+1}^{\prime}=\cdots=\beta_{b+a}^{\prime}=\beta_{b}$. (This is closely related to rules (iv) and (ii) of $\S 4$.)

(N1a) If $c>b$ and $\beta_{c}=0$, then $N_{b, c}(\beta ; z f, f)=N_{b, c-1}(\beta ; z f, f)$.

(N1b) If $c=b$ and $\beta_{c}=-1$, then $N_{b, c}(\beta ; z f, f)=0$. 
(N2a) If $b=0$ and $\beta_{1}=n+1$, then $N_{b, c}(\beta, z f, f)=N_{1, c}\left(\beta^{\prime} ; z f, f\right)$, where $\beta_{1}^{\prime}=n$.

(N2b) If $b \geq 1$ and $\beta_{1}=n+1$, then $N_{b, c}(\beta ; z f ; f)=0$.

Proof. For (N0) apply $f_{k}(x, z)=f_{k}(x)+z f_{k-1}(x)$ in columns $b+1$ through $b+a$. Expand into $2^{a}$ determinants with respect to these two part columns, and use (GM) to see that only the term with $\beta_{b+1}^{\prime}=\cdots=\beta_{b+a}^{\prime}=\beta_{b}$ is nonzero. For rule (N1a), note that the last column has only a " 1 " in the last row. For rule $(\mathrm{N} 1 \mathrm{~b})$, the last column is entirely " 0 "s. The proof of $(\mathrm{N} 2 \mathrm{~b})$ is the same as (M2) of Lemma 8.1. For (N2a) apply $f_{k}(x, z)=f_{k}(x)+z f_{k-1}(x)$ to the first column and again use the proof of (M2).

Proof of Theorem 9.1. For part (a) replace $\mathrm{sp}_{\lambda, \lambda}(y)$ with

$$
\operatorname{sp}_{\mu}(y)=M_{c}\left(\beta ; f\left(y, y^{-1}\right)\right) .
$$

Use Lemma 9.2 with $u=f_{1}(x)=x_{1}+\cdots+x_{2 n}$. Then for $f_{1} M_{c}(\beta ; f)$ we get $M_{c+1}\left(\beta, \beta_{c+1}^{\prime}=1 ; f\right)$ plus the two sums describing the possibilities of adding or removing a square to any existing column. If the resulting $\beta_{j}^{\prime}$ sequence is not weakly decreasing, then by (GM) that term is 0 . If the last column is decreased to zero length, then (M1) redefines the size of the determinant. Finally, specialize $x \mapsto y$ on both sides. By (M2) any terms $M_{c}\left(\beta^{\prime} ; f\right)$ with $\beta_{i}^{\prime}=n+1$ will vanish.

For part (b) replace $\operatorname{sp}_{\lambda, \mu}(y ; z)$ with

$$
\mathrm{sp}_{\mu, b}(y, z)=N_{b, c}\left(\beta ; z f\left(y, y^{-1}\right), f\left(y, y^{-1}, z\right)\right) .
$$

Use Lemma 9.2 with $u=f_{1}(x, z)=x_{1}+\cdots+x_{2 n}+z$. By (GM) only terms $N_{b, c}\left(\beta^{\prime} ; h, g\right)$ with $\beta_{j}^{\prime} \geq \beta_{j+1}^{\prime}$ for $1 \leq j \leq c-1$ except $j=b$ survive. In either of the two sums it is possible for a term to arise with $\beta_{b}^{\prime}<\beta_{b+1}^{\prime}=\beta_{b}^{\prime}+1$ in the same manner as in cases (ii) or (iv) of $\S 4$. Here

$$
\beta_{b+1}^{\prime}=\beta_{b+2}^{\prime}=\cdots=\beta_{b+a}^{\prime}=\beta_{b}^{\prime}+1,
$$

for some $a \geq 1$. Apply rule (N0) to obtain output terms with additional marked squares as according to rules (ii) and (iv). Otherwise we have $\beta_{b}^{\prime} \geq \beta_{b+1}$ at the outset. The change of one $\beta_{j}$ by +1 or -1 corresponds to adding or removing one unmarked square as in rules (i) or (iii) respectively. If there is just one square in the last column, consider the term resulting from decrementing the last column of the determinant. If the square was unmarked, then rule (N1a) redefines the determinant size. If the square was marked, rule (N1b) prohibits its removal. Again specialize $x \mapsto y$ on both sides. The rule (N2a) will mark the last square of the first column in a term where it has just grown to length $n+1$; and the rule (N2b) will kill a term where the first column has just grown to total length $n+2$. 


\section{REFERENCES}

[Be1] A. Berele, A Schensted-type correspondence for the symplectic group, J. Combin. Theory Ser. A 43 (1986), 320-328.

[Be2] - A construction of Sp-modules by tableaux, Linear and Multilinear Algebra 19 (1986), 299-307.

[Boe] H. Boerner, Representations of groups, North-Holland, Amsterdam, 1963.

[G-V] I. Gessel and G. Viennot, Determinants, paths, and plane partitions, in preparation.

[H-W] P. Hanlon and D. Wales, On the decomposition of Brauer's centralizer algebras, J. Algebra 121 (1989), 409-445.

[Hum] J. Humphreys, Introduction to Lie algebras and representation theory, Springer-Verlag, New York, 1972.

[Ki1] R. C. King, Weight multiplicities for the classical groups, Group Theoretical Methods in Physics, (Janner, Janssen, and Boon, eds.), Lecture Notes in Physics, vol. 50, SpringerVerlag, New York, 1976.

[Ki2] _ Modification rules and products of irreducible representations of the unitary, orthogonal, and symplectic groups, J. Math. Phys. 12 (1971), 1588-1598.

[K-T] K. Koike and I. Terada, Young-diagrammatic methods for the representation theory of the classical groups of type $B_{n}, C_{n}, D_{n}$, J. Algebra 107 (1987), 466-511.

[Lit] D. E. Littlewood, The theory of group characters, 2nd ed., Oxford Univ. Press, London, 1950.

[Mac] I. G. Macdonald, Symmetric functions and Hall polynomials, Oxford Univ. Press, Oxford, 1979.

[Pr1] R. Proctor, Odd symplectic groups, Invent. Math. 92 (1988), 307-332.

[Pr2] _ Four Littlewood-Richardson proofs, unpublished notes.

[Pr3] _ A Schensted algorithm which models tensor representations of the orthogonal group, Canad. J. Math. 42 (1990), 28-49.

[Pr4] _ Young tableaux, Gelfand patterns, and branching rules for classical groups, preprint.

[R-W] A. Ram and H. Wenzl, Matrix units for centralizer algebras, preprint.

[Sch] C. Schensted, Longest increasing and decreasing subsequences, Canad. J. Math. 13 (1961), 179-191.

[Sun] S. Sundaram, On the combinatorics of representations of $\operatorname{Sp}(2 n, \mathbf{C})$, Ph.D. Thesis, M.I.T., 1986.

[Tho] G. P. Thomas, On a construction of Schützenberger, Discrete Math. 17 (1977), 107-118.

[Wey] H. Weyl, The classical groups, Princeton Univ. Press, Princeton, N.J., 1939.

[Zhe] D. P. Zhelobenko, The classical groups. Spectral analysis of their finite dimensional representations, Russian Math. Surveys 17 (1962), 1-94.

Department of Mathematics, University of California, Los ANgeles, California 90024

Current address: Department of Mathematics, University of North Carolina, Chapel Hill, North Carolina 27599 